\title{
Subgroup Discovery in Structural Equation Models
}

\author{
Christoph Kiefer ${ }^{1}$, Florian Lemmerich ${ }^{2}$, Benedikt Langenberg ${ }^{1}$, and Axel Mayer ${ }^{1}$ \\ ${ }^{1}$ Bielefeld University, Bielefeld, Germany \\ ${ }^{2}$ University of Passau, Passau, Germany
}

(O2022, American Psychological Association. This paper is not the copy of record and may not exactly replicate the final, authoritative version of the article. Please do not copy or cite without authors' permission. The final article will be available, upon publication, via its DOI: $10.1037 / \operatorname{met} 0000524$

\begin{abstract}
Author Note
Correspondence concerning this article should be addressed to Christoph Kiefer, Methods and Evaluation, Department of Psychology, Bielefeld University, Universitätsstraße 25, D-33501 Bielefeld, Germany, e-mail: christoph.kiefer@uni-bielefeld.de This work was supported by the Excellence Initiative of the German federal and state governments (RWTH PrepFund project).

This paper uses data from the National Educational Panel Study (NEPS): Starting Cohort Grade 5, doi:10.5157/NEPS:SC3:10.0.0. From 2008 to 2013, NEPS data was collected as part of the Framework Program for the Promotion of Empirical Educational Research funded by the German Federal Ministry of Education and Research (BMBF). As of 2014, NEPS has been carried out by the Leibniz Institute for Educational Trajectories (LIfBi) at the University of Bamberg in cooperation with a nationwide network.
\end{abstract}




\begin{abstract}
Structural equation modeling (SEM) is one of the most popular statistical frameworks in the social and behavioural sciences. Often, detection of groups with distinct sets of parameters in structural equation models (SEM) are of key importance for applied researchers, for example, when investigating differential item functioning for a mental ability test or examining children with exceptional educational trajectories. In this paper, we present a new approach combining subgroup discovery - a well-established toolkit of supervised learning algorithms and techniques from the field of computer science - with structural equation models termed SubgroupSEM. We provide an overview and comparison of three approaches to modeling and detecting heterogeneous groups in structural equation models, namely, finite mixture models, SEM trees, and SubgroupSEM. We provide a step-by-step guide to applying subgroup discovery techniques for structural equation models, followed by a detailed and illustrated presentation of pruning strategies and four subgroup discovery algorithms. Finally, the SubgroupSEM approach will be illustrated on two real data examples, examining measurement invariance of a mental ability test and investigating interesting subgroups for the mediated relationship between predictors of educational outcomes and the trajectories of math competencies in 5th grade children. The illustrative examples are accompanied by examples of the $\mathrm{R}$ package subgroupsem, which is a viable implementation of our approach for applied researchers.

Keywords: structural equation modeling, exceptional model mining, subgroup discovery, exploratory data mining
\end{abstract}




\section{Subgroup Discovery in Structural Equation Models}

The investigation and modeling of groups with distinct sets of parameters (hereafter: heterogeneous groups) in structural equation models (SEM) has a long tradition in the social and psychological sciences. In an 'ordinary' single-group SEM, it is assumed that a single set of parameter values is sufficient to describe the relations among the variables. However, it is often more plausible that different groups of individuals exhibit different sets of parameter values. Fifty years ago, Jöreskog (1971) proposed the multi-group SEM framework, which was the first to allow simultaneous estimation of a SEM with multiple (known) groups, estimating different sets of parameter values for different groups. Further developments in this direction have made it possible to examine the effect of unobserved/unknown groups by inferring group membership from observed variables in the model or auxiliary variables. Prominent examples are the MIMIC approach (Muthén, 1989) and the finite mixture extensions of SEM Arminger, Stein, \& Wittenberg, 1999; Jedidi, Jagpal, \& DeSarbo, 1997).

In recent years and with the rise of modern computer technology, data mining and machine learning techniques from computer science have been adapted to efficiently unravel heterogeneity in structural equation models fitted on datasets from large-scale assessment studies. In large datasets, unknown groups might potentially be formed based on a multitude of variables, making it a crucial task to discriminate potentially interesting groups from irrelevant ones. Based on a decision tree paradigm, a structural equation model tree (SEM tree; Brandmaier, von Oertzen, McArdle, \& Lindenberger, 2013) can be used to identify relevant groups by recursively partitioning the covariate space in the way that most improves overall model fit. That is, the dataset is split based on a covariate to obtain the two groups that provide the best model fit. Then, the two groups are each again split based on another covariate and so on until the covariate space is completely partitioned into structurally distinct groups and no further model fit improvements can be achieved (for an introduction to recursive partitioning, see Strobl, Malley, \& Tutz, 2009). 
However, the main goal of structural equation model trees is to precisely predict distinct groups and group memberships, which sometimes comes at the cost of interpretability of the results, especially if an ensemble of SEM trees (known as a SEM forest; Brandmaier, Prindle, McArdle, \& Lindenberger, 2016) are employed to stabilize the predictive performance.

In this paper, we present an alternative approach to investigating heterogeneous groups in structural equation models termed SubgroupSEM, which builds on algorithms from subgroup discovery and its extension, exceptional model mining (Klösgen, 1996; Leman, Feelders, \& Knobbe, 2008). In contrast to decision trees, subgroup discovery does not primarily focus on prediction accuracy but on identifying interesting subset patterns with human-interpretable group descriptions. Two key features of the SubgroupSEM approach are (a) the possibility to detect groups based on an user-defined "interestingness measure" (IM) and (b) its computational efficiency, building on state-of-the-art techniques from computer science. These make it a powerful tool for the tailored exploration of heterogeneous groups within large datasets.

SubgroupSEM has recently been applied to find heterogeneous groups in mediation models (Lemmerich, Kiefer, Langenberg, Cacho Aboukhalil, \& Mayer, 2020) and latent growth models (Mayer, Kiefer, Langenberg, \& Lemmerich, 2021). Both papers provide a brief introduction to special cases of SEM (i.e., mediation models, latent growth curve models) and showcase the use of subgroup discovery in these restricted scenarios with applied examples. However, they lack explanations for how the approach generalizes to the broader class of structural equation models and do not provide details on the algorithmic procedures underlying SubgroupSEM. In addition, neither paper provides a detailed comparison to alternative methods, like finite mixture models or SEM trees, or guidance for applied researchers on how to employ the approach in statistical software environments such as R.

This paper presents in-depth the subgroup discovery approach in general cases of 
structural equation modeling and is structured as follows: We first provide an overview and illustrated comparison of three approaches to modeling and detecting heterogeneous groups in structural equation models, namely, finite mixture models, SEM trees, and

SubgroupSEM. In addition, we offer guidance to researchers on selecting a method. Then, we provide a step-by-step guide to applying subgroup discovery techniques to structural equation models in general. This is followed by a detailed, illustrated presentation of pruning strategies and four subgroup discovery algorithms. Finally, the SubgroupSEM approach will be illustrated on two real data examples, with $\mathrm{R}$ procedures for implementing the method described. The examples illustrate how interesting subgroups can be investigated (a) in a confirmatory factor analysis of a mental ability test and (b) in a partially mediated latent growth model with multiple indicators and mediators from a large-scale educational assessment study.

\section{Heterogeneous Groups in Structural Equation Models}

Structural equation models are a popular and powerful tool in the social sciences. They are employed for confirmatory factor analysis within classical test theory or latent state-trait theory (Steyer, Mayer, Geiser, \& Cole, 2015), allow for path analysis (e.g., mediation models) with latent variables (MacKinnon, 2012), and are frequently used to analyze longitudinal data, for example, using latent growth curve models (McArdle, 1988. Meredith \& Tisak, 1990). In a conventional (single-group) SEM, it is assumed that all observations in the data are drawn from a single population and that the estimated set of parameters holds equally for all groups within that population. However, this assumption may be too restrictive in many cases where heterogeneous groups do indeed exist - for example, if a mediating process works differently for female and male students, or if a psychometric test systematically disadvantages a certain subgroup.

Thus, it is often necessary to fit a SEM to several groups which may differ with regard to their model parameters. The general form of a multi-group SEM with either observed or unobserved group membership $g=1, \ldots, h$ on the group variable $G$ is defined 
according to a group-specific measurement model relating a vector of manifest variables $\mathbf{y}$ to a vector of latent variables $\boldsymbol{\eta}$ and a group-specific structural model specifying structural relations among latent variables $\boldsymbol{\eta}$ :

$$
\begin{array}{lr}
\mathbf{y}=\boldsymbol{\nu}_{g}+\boldsymbol{\Lambda}_{g} \boldsymbol{\eta}+\boldsymbol{\varepsilon} & \text { Measurement model } \\
\boldsymbol{\eta}=\boldsymbol{\alpha}_{g}+\mathbf{B}_{g} \boldsymbol{\eta}+\boldsymbol{\zeta} & \text { Structural model }
\end{array}
$$

where $\boldsymbol{\nu}_{g}$ is a group-specific vector of measurement intercepts; $\boldsymbol{\Lambda}_{g}$ is a group-specific matrix of loadings; $\boldsymbol{\alpha}_{g}$ is a group-specific vector of structural intercepts; $\mathbf{B}_{g}$ is a group-specific matrix of structural coefficients; $\varepsilon$ is a vector of measurement error variables with zero mean vector and group-specific covariance matrix $\Theta_{g} ; \boldsymbol{\zeta}$ is a vector of structural residuals with zero mean vector and group-specific covariance matrix $\Psi_{g}$. As all vectors and matrices indexed with a $g$ can vary among groups, the multi-group approach provides the opportunity to compare groups with regard to measurement and structural parameters.

In traditional multi-group structural equation modeling (Jöreskog, 1971, Sörbom, 1974), the researcher specifies the group variable $G$ of interest in order to compare measurement and structural parameters among groups. For example, a researcher could investigate differences in boys' and girls' educational trajectories by estimating a latent growth curve model (Meredith \& Tisak, 1990) for each sex within the multi-group framework. If these groups are indeed heterogeneous, accounting for the distinct parameter sets using a multi-group model will likely lead to an improved model fit (compared to a model with equality constraints across groups).

\section{Introductory example}

In applied settings it is often unknown which particular groups have a distinct set of parameters, especially when examining complex relationships and large datasets (and therefore many potential groups). Below, we provide an overview of popular approaches to identifying heterogeneous subgroups within structural equation models. Using an artifical 
example, we will review the similarities and differences between finite mixture modeling, decision trees and subgroup discovery.

Consider a hypothetical dataset of $N=5000$ pupils uniformly drawn from five schools (variable $A$ ) and across five grades (variable $B$ ). We simulated a single-factor model as illustrated in Figure 11 (i.e., one factor measured by five indicators), which could reflect a math competency test with particular focus on geometry. ${ }^{1}$ While the measurement model was invariant for the majority of pupils, we varied the model parameters for three specific groups, namely: group $G_{1}$ consisting of pupils from school $A=3$ (e.g., the geometry school, with special focus on geometry across all grades); group $G_{2}$ consisting of pupils from grade $B=1$ (e.g., the grade, where geometry is always part of the curriculum); and group $G_{3}$ consisting of pupils from grade $B=5$ at school $A=5$ (e.g., whose teacher is not particularly adept at geometry). Detailed information about the group-specific parameters are provided in Table 1. The upper left panel of Figure 2 illustrates the covariate space of $A$ and $B$ and the positioning of groups $G_{1}, G_{2}$, and $G_{3}$ therein. As this grouping is usually not known beforehand, we can apply different techniques to try to detect it. Below, we explain and apply the finite mixture framework, structural equation model trees and SubgroupSEM.

\section{Finite Mixture-Based SEM}

Finite mixture-based SEM (Arminger et al., 1999, Jedidi et al., 1997), also called structural equation mixture modeling (SEMM), integrates a finite mixture approach (McLachlan \& Peel, 2000) with the SEM framework. The concept of specifying group-specific models is similar to multigroup SEM, with the difference that the group weights as well as group memberships are unobserved and have to be estimated from the data. Special cases of SEMM include growth mixture models (Muthén \& Muthén, 2000) to model differential trajectories, latent class analysis (Collins \& Lanza, 2013), and factor

\footnotetext{
1 The data simulation and analysis script (i.e., R scripts) for all three approaches as well as the simulated dataset are available in the online supplementary material.
} 
Table 1

Parameters for the measurement model of heterogenous groups in the introductory example

\begin{tabular}{|c|c|c|c|c|}
\hline Parameter & Initial & $G_{1}$ & $G_{2}$ & $G_{3}$ \\
\hline \multicolumn{5}{|c|}{ Factor loadings } \\
\hline$\lambda_{1}$ & 1.0 & 1.0 & 1.0 & 1.0 \\
\hline$\lambda_{2}$ & 0.9 & 1.1 & 1.1 & 0.7 \\
\hline$\lambda_{3}$ & 0.8 & 1.2 & 1.2 & 0.6 \\
\hline$\lambda_{4}$ & 0.7 & 1.3 & 1.3 & 0.5 \\
\hline$\lambda_{5}$ & 0.6 & 1.4 & 1.4 & 0.4 \\
\hline \multicolumn{5}{|l|}{ Intercepts } \\
\hline$\nu_{1}$ & 0.0 & 0.0 & 0.0 & 0.0 \\
\hline$\nu_{2}$ & 0.1 & 0.1 & 0.1 & -0.1 \\
\hline$\nu_{3}$ & 0.2 & 0.2 & 0.2 & -0.2 \\
\hline$\nu_{4}$ & 0.3 & 0.3 & 0.3 & -0.3 \\
\hline$\nu_{5}$ & 0.4 & 0.4 & 0.4 & -0.4 \\
\hline \multicolumn{5}{|c|}{ Residual variances } \\
\hline $\operatorname{Var}\left(\varepsilon_{1}\right)$ & 1.0 & 1.0 & 1.0 & 1.2 \\
\hline $\operatorname{Var}\left(\varepsilon_{2}\right)$ & 1.0 & 1.0 & 1.0 & 1.2 \\
\hline $\operatorname{Var}\left(\varepsilon_{3}\right)$ & 1.0 & 1.0 & 1.0 & 1.2 \\
\hline $\operatorname{Var}\left(\varepsilon_{4}\right)$ & 1.0 & 1.0 & 1.0 & 1.2 \\
\hline $\operatorname{Var}\left(\varepsilon_{5}\right)$ & 1.0 & 1.0 & 1.0 & 1.2 \\
\hline
\end{tabular}

Note. The measurement model for the whole sample was initially simulated with the values given in column Initial. Bold values indicate, which parameters were subsequently varied in groups $G_{1}, G_{2}$, and $G_{3}$.

mixture modeling (Lubke \& Muthén, 2005). In recent years, the SEMM framework has been extended to account for non-linear relationships and non-normal latent distributions (Kelava, Nagengast, \& Brandt, 2014) as well as Bayesian estimation and analysis (Liu \& Song, 2018).

In SEMM, the heterogeneous groups are not directly observed, but implied by fitting a mixture of distributions to the observed data. The group variable is referred to as latent class $C$ and the number $N_{c}$ of latent classes (i.e., groups) must be predetermined. For example, when investigating educational trajectories, the researcher can assume that $N_{c}$ groups with differential trajectories exist and estimate a growth mixture model for them. In practice, the number of latent classes is usually determined by fitting the model with a mixture of $1,2, \ldots, N_{c}, \ldots$ distributions and then choosing the number which provides the best model fit (e.g., using fit indices or a likelihood ratio test). The results of 


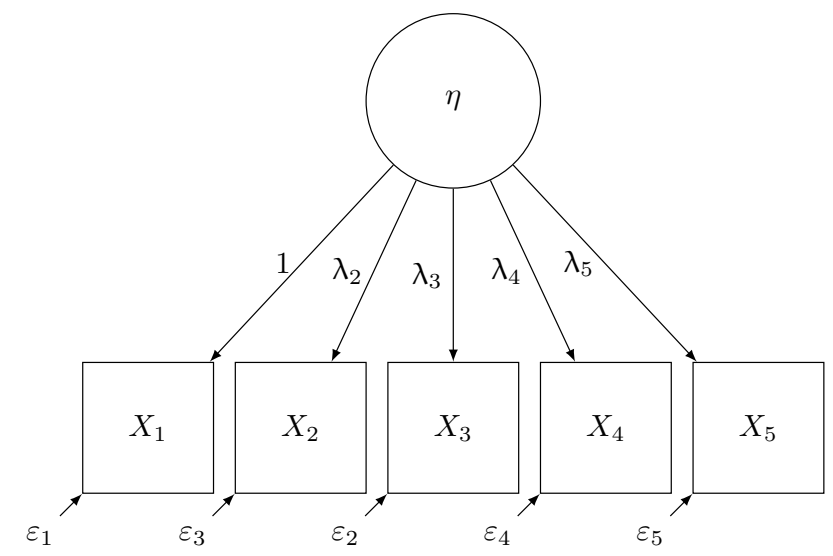

Figure 1

Path model of single-factor model (i.e., latent variable $\eta$ ) measured by five indicators (i.e., manifest variables $X_{1}-X_{5}$ ) for introductory example

the chosen model commonly include parameter estimates as well as the estimated proportions of the latent classes. Additionally, for each person in the sample, the probability of being in one latent class or another can be obtained.

Often, mixture models are seen as "elegant procedures" (see Steinley \& Brusco, 2011, p. 63, providing an overview of similarities and differences between mixture modeling and traditional clustering techniques) because they incorporate the probabilistic nature of modeling an unobservable group structure. However, this probabilistic nature also comes with some downsides for exploratory research: First, the detection of latent classes is based on the observed variables included in the model alone. That is, additional variables explaining the group structure and allowing for a substantive interpretation of the groups have to be examined in an additional step (cf. Lanza, Tan, \& Bray, 2013). Second, even examining the latent class with regard to additional variables does not necessarily result in finding useful patterns. As persons are not deterministically assigned to the classes, we cannot be sure whether observing many persons with the same covariate values and high probability for a specific latent class is a substantive pattern or not. Third, mixture models can be difficult to estimate, as the log-likelihood function often has multiple local maxima that prevent the optimizer from finding the global maximum, thus leading to faulty results 

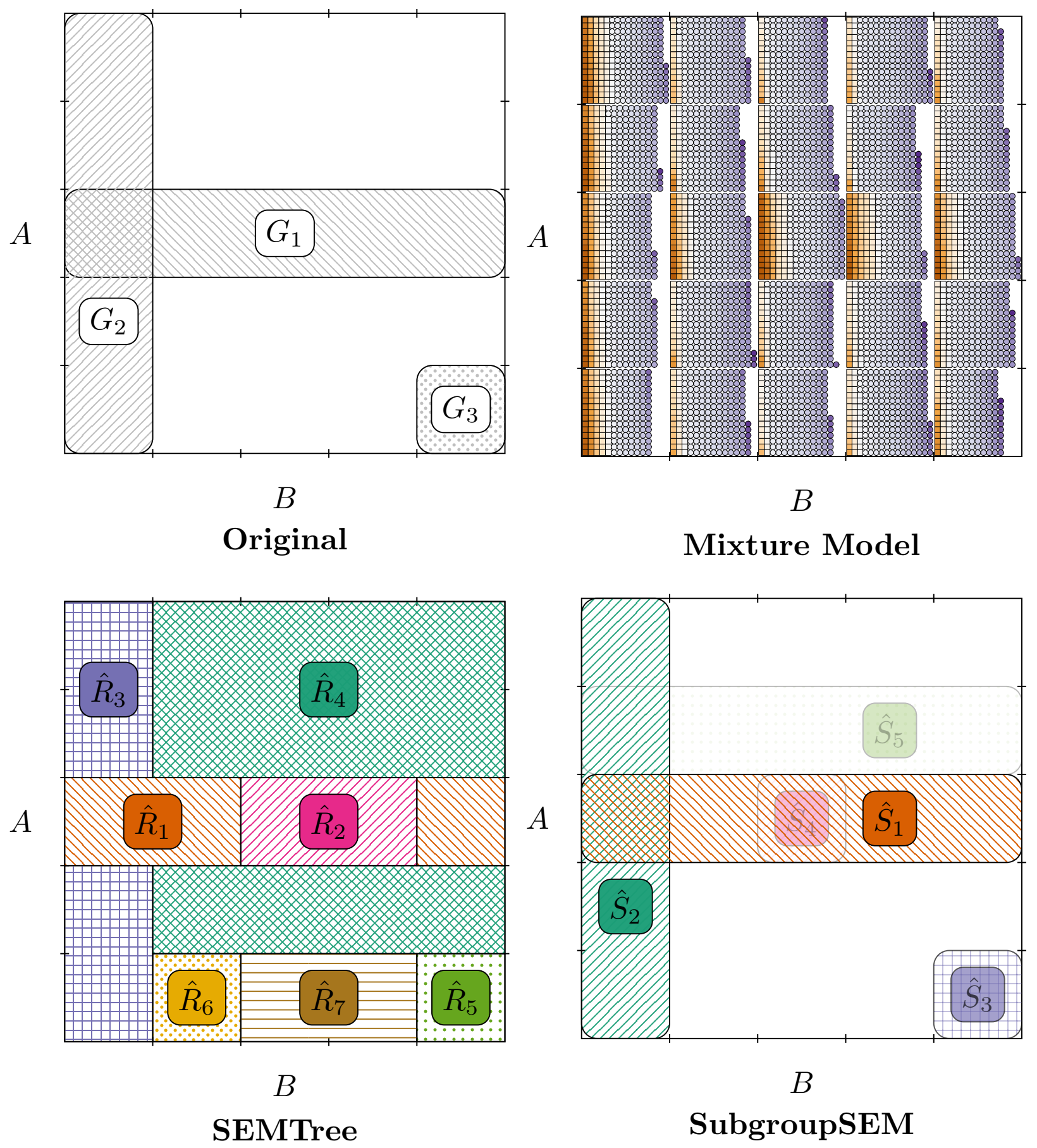

Figure 2

Graphical comparison of approaches to detect heterogeneous subgroups. Upper left panel shows for which areas of the covariates $A$ and $B$ were simulated with distinct sets of parameters. Upper right panel illustrates a two-class solution of the SEMM, where each person is assigned to either class $C=1$ (rectangles) or $C=2$ (circles) with an individual probability (color intensity). Lower left panel shows a decision tree map (i.e., SEMTree), where seven non-overlapping areas $R_{i}$ with distinctive sets of model parameters are identified (indicated by colors and patterns). The covariate space is fully partitioned. Lower left panel shows a subgroup discovery map (i.e., selected subgroups, partially overlapping and not classifying the whole covariate space). $S_{i}$ denotes the subgroups from subgroup discovery, where color intensity indicates the interestingness of the subgroup. 
(Hipp \& Bauer, 2006; Shireman, Steinley, \& Brusco, 2016). (See Heath, Fu, \& Jank, 2009 Shireman, Steinley, \& Brusco, 2017, for strategies for dealing with estimation issues.)

Applying a finite mixture-based SEM to our introductory example, we find support for a two class solution. The parameter estimates in the two-class solution resemble the parameters for the overall group (i.e., class 2) and the parameters for the manipulated groups $G_{1}$ and $G_{2}$ (i.e., class 1 ). The model assigns each unit a probability of being either in class 1 (i.e., rectangles) or class 2 (i.e., circles), which we plotted against our covariates (i.e., school $A$ and grade $B$ ) in the upper right panel of Figure 2 (stronger color indicating a higher probability of being in the respective class). While most units with a high probability of class 1 seem be found in the areas of the manipulated groups $G_{1}$ and $G_{2}$, the majority of units in this range would still be assigned to class 2 . Thus, these findings suggest that two heterogeneous groups can be distinguished, but we cannot safely conclude how these can be interpreted substantively. In addition, the manipulated group $G_{3}$ would remain undetected with this approach.

\section{Decision Trees}

From the perspective of data mining, the search for heterogeneous groups with respect to a specific property can be approached as a supervised learning task comparable to classification. That is, we are looking for manifest groupings based on a set of covariates (e.g., female participants over age 40) for which the true set of parameters is different from the rest of the population. The advantage of data mining techniques is that although the groups are unknown, a manifest, rule-based, easily interpretable group description can be provided for each group - in contrast to finite mixture models. Common techniques for classification tasks are decision trees and association rules. For an overview of applications of machine learning techniques in the behavioral sciences, see Kliegr, Bahník, and Fürnkranz 2020).

The decision tree paradigm Quinlan, 1986) dates back to early works on automated interaction detection (Morgan \& Sonquist, 1963) and has been extended to a variety of 
model classes (cf. Zeileis, Hothorn, \& Hornik, 2008). In decision tree-based approaches to finding heterogeneous groups in SEM, like SEM Tree (Brandmaier et al., 2013), the group variable $G$ is generated from a set of covariates by fully partitioning the covariate space into a finite number of subsets. SEM trees have been applied, for example, in neurocognitive research (de Mooij, Henson, Waldorp, \& Kievit, 2018; Simpson-Kent et al., 2020), in clinical psychology (Ammerman, Jacobucci, \& McCloskey, 2019), and developmental psychology (Brandmaier, Ram, Wagner, \& Gerstorf, 2017). Besides the semtree package from Brandmaier et al. (2013), it is possible to estimate SEM trees with the partykit package (Hothorn \& Zeileis, 2015) within the R environment, or by using an $\mathrm{R}$ interface to Mplus (Serang et al., 2021).

Structural equation model trees are constructed via recursive partitioning of the covariate space. The procedure starts with the whole sample (single-group model) and iteratively compares it to dichotomous splits of the covariates (two group model; e.g., for male and female participants). The split leading to the best improvement in model fit (e.g., tested with a likelihood ratio test) is chosen as the next tree node. The procedure is then repeated recursively within the partitions identified in the previous step until a stopping criterion is reached. For example, if gender was chosen as the tree node in the first step, the algorithm would proceed by comparing female participants (single-group model) with further splits (e.g., women under 30 vs. women over 30). As a result, a decision tree is created with leaves indicating subgroups of the sample with a distinct set of parameter estimates. For an introduction to recursive partitioning, see Strobl et al. (2009).

One particular goal and strength of decision tree approaches lies in predictive modeling, that is, inferring a classification scheme that provides useful information to predict performance or behavior in future datasets. However, this strength can also be considered as an Achilles' heel, when it comes to generating hypotheses about potentially interesting and interpretable groups for future research. First, potential groups must be strictly disjoint (i.e., no overlap is possible). This can be a drawback in an exploratory 
search for groups as an early partitioning decision cannot be undone later in the process. For example, if a decision tree introduces an early split between female and male participants, each subsequent split automatically keeps the separation between these two groups - however, if all persons under the age of 30 compared to persons over 30 would be almost as interesting as the gender split, there would be no chance of observing this split within our decision tree. Second, the process results in a number of tree leaves or a fully partitioned covariate space, with no indication which of the groups might be the most interesting or exceptional one. For example, if we examine an educational dataset for groups of students at high risk for negative educational outcomes, a decision tree would provide us with a classification of risk profiles for the whole covariate space, but not any immediate information about which of these groups has the highest risk.

Applying a SEM tree to our introductory example yields the partitioning of the covariate space illustrated in the lower left panel of Figure 2. We find a total of seven tree leaves, which partially correspond to the originally manipulated groups, but with some additional splits introduced. An early split for $A=3$ (corresponding to group $G_{1}$ ) prevents fully identifying the overlapping group $G_{2}$ as a whole and $A=3$ is later split into two

groups $\hat{R}_{1}$ and $\hat{R}_{2}$ (dividing $G_{1}$ in two distinct partitions). Group $G_{3}$ is correctly identified as partition $\hat{R}_{5}$. Note that there is no immediate information about whether all of these partitions are equally interesting or some partitions are exceptionally different from the others. However, this information can be obtained by manually inspecting the individual leaves, which can be tedious for larger trees.

\section{Subgroup Discovery}

An alternative approach addressing the Achilles' heel of decision trees is the field of subgroup discovery (Herrera, Carmona, González, \& del Jesus, 2011; Klösgen, 1996) from computer science. The primary goal of subgroup discovery lies not in prediction, but in identifying interesting and understandable patterns with respect to the selected target concept. Some of the pioneering algorithms in this field are actually extensions of the 
decision tree paradigm that focus on increasing human understandability of the identified subgroups (Herrera et al., 2011) Subgroup discovery has been successfully applied, for example, in medicine to detect risk groups for coronary heart disease Gamberger, Lavrač, \& Krstačić, 2003) or in bioinformatics to identify subgroups of leukemia ALL cancer (Trajkovski, Zelezny, Lavrac, \& Tolar, 2008). For further real-world problems solved through subgroup discovery, see Herrera et al. (2011). An important extension of subgroup discovery is the field of exceptional model mining (Duivesteijn, Feelders, \& Knobbe, 2016, Leman et al., 2008), which applies subgroup discovery algorithms to investigate heterogeneous groups in statistical models. In recent years, exceptional model mining has been developed for survival analysis (Mattos, Silva, de Mattos Neto, \& Vimieiro, 2020), regression models (Duivesteijn, Feelders, \& Knobbe, 2012), and mediation models (Lemmerich et al., 2020), to name a few examples.

Key advantages of subgroup discovery and exceptional model mining over the classical decision tree paradigm are fourfold: First, the "interestingness" of a subgroup is defined by the researcher via a so called interestingness measure (IM). For example, if a researcher is interested in subgroups with strong indirect effects within a simple mediation model, the IM can be the magnitude of the indirect effects within each subgroup. Thus, the IM interactively helps to tailor the subgroup discovery task to the researchers interests. Second, the search space is not necessarily fully partitioned, but only subgroups of high interest are returned. Thus, not every value combination of the covariates ends up being an "interesting result"; only value combinations representing exceptional parameter constellations are presented to the researcher. Third, partly overlapping subgroups are possible, including aggregations of smaller subgroups. In our age and gender example above, subgroup discovery would be able to separate female from male participants as well as participants under 30 years old from over 30 years old, which exhibit similar

\footnotetext{
${ }^{2}$ In the field, the term subgroup is used to refer to a subset of the population. The term can be understood interchangeably with our previously used term of group.
} 
interestingness, even though these groups overlap. Fourth, subgroup discovery includes a number of algorithms and strategies to explore large covariate spaces with reasonable computational demand. While some algorithms are exhaustive and guarantee an optimal solution, others are heuristic and more easily scalable to larger datasets. We give a more detailed overview of these algorithms and strategies later in the paper.

We briefly anticipate the results of SubgroupSEM in our introductory example to illustrate how the approach differs conceptionally from finite mixture-based SEM and SEM trees. For this application, likelihood-ratio test statistics and an exhaustive search algorithm are used to try to detect the originally manipulated groups $G_{1}, G_{2}$, and $G_{3}$. The likelihood-ratio test statistic serves as a measures of exceptionality or interestingness measure of a subgroup compared to the rest of the sample. As you can see in the lower right panel of Figure 2, the original groups $G_{1}, G_{2}$, and $G_{3}$ are correctly identified as the three most interesting subgroups, namely $\hat{S}_{1}, \hat{S}_{2}$, and $\hat{S}_{3}$. Spurious findings and less

interesting groups such as $\hat{S}_{4}$ and $\hat{S}_{5}$ can be identified by their low interestingness measures (illustrated by color intensity).

In the following section, we provide a step-by-step guide to how subgroup discovery can be applied to structural equation models and explain the central concepts of subgroup discovery. In the section thereafter, we provide more details on the algorithmic components of a subgroup discovery task as well as on our software implementation of SubgroupSEM.

\section{Subgroup Discovery in Structural Equation Models}

A subgroup discovery task is usually defined by a target concept, a search space, a quality or interestingness measure, and a search strategy. These four concepts also reflect the steps necessary to specify and conduct a subgroup discovery task in SEM, which we call SubgroupSEM. Thus, we will walk through these four concepts step-by-step, explaining how they can be applied to detect heterogeneous groups and mine exceptional structural equation models. Listing 1 provides a comprehensive overview of how these four steps can be run in $\mathrm{R}$ using the subgroupsem package. A substantive illustration using $\mathrm{R}$ code is 


\section{Table 2}

Summary table comparing techniques of identifying heterogeneous subgroups within structural equation models

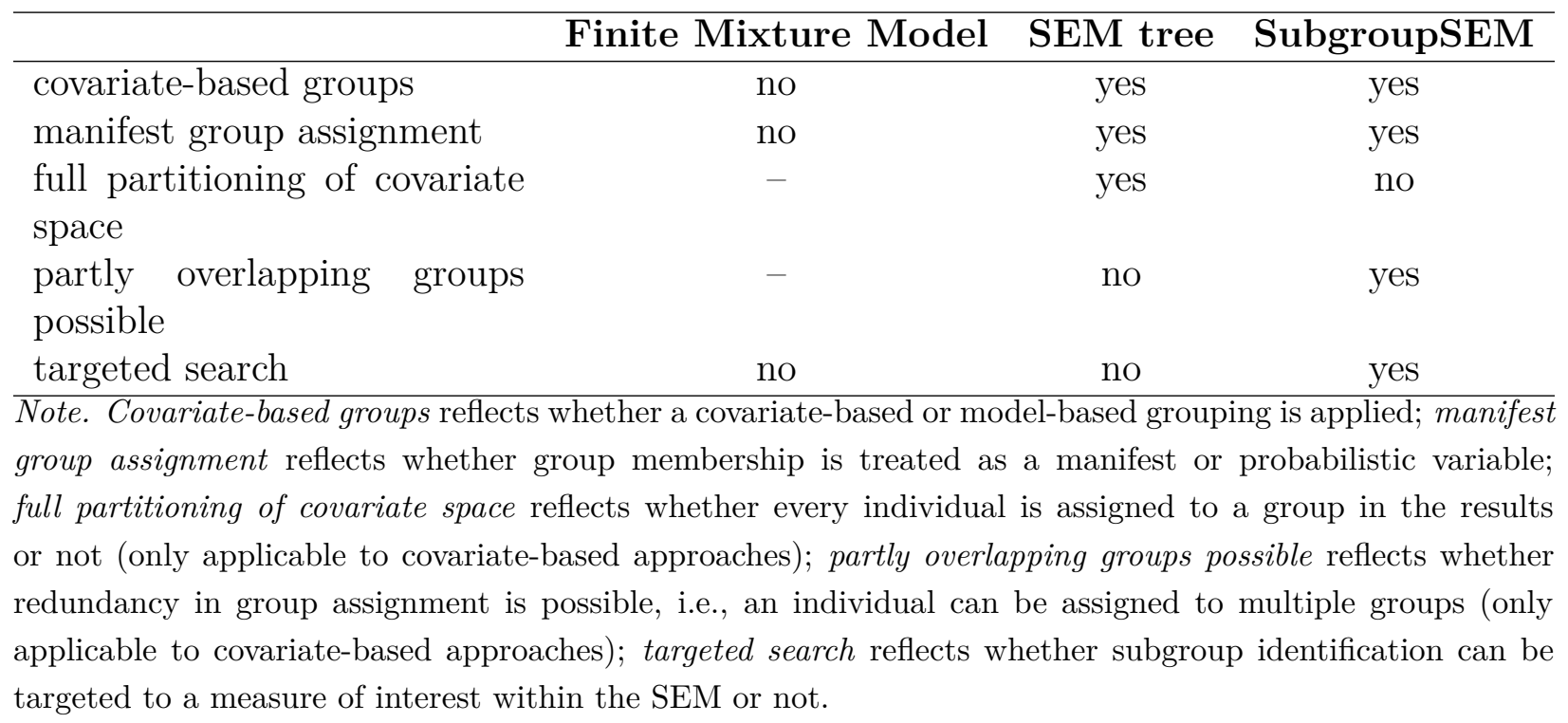

given in the Illustrative Examples section.

\section{Step 1: Specifying a Structural Equation Model or: The Target Concept}

The target concept $T$ defines the property, parameter, or model of interest for a subgroup discovery task. In exceptional model mining, it is a model fitted for each subgroup. It features a model, which is fixed for a specific mining task (e.g., a structural equation model), and parameter values, which depend on the estimated model in the respective subgroup. The goal of exceptional model mining is to identify subgroups for which the model parameters differ considerably from the parameters of the model built from the entire dataset or from the complement of the subgroup.

For SubgroupSEM, a two-group structural equation model is used as the model 


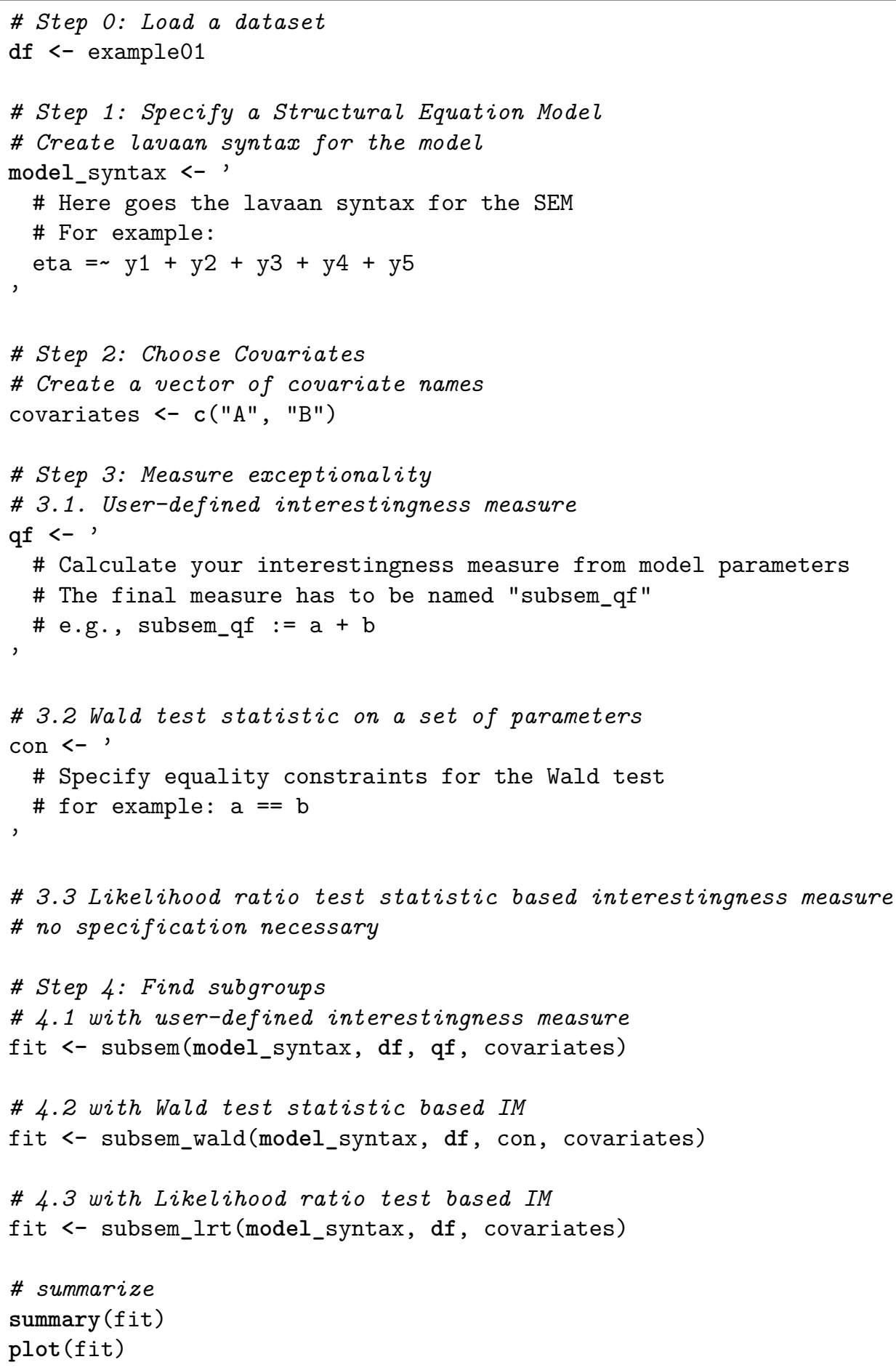

Listing 1: Steps to conduct subgroup discovery in SEM using the subgroupsem package 
class:

\begin{tabular}{cr}
$\mathbf{y}=\boldsymbol{\nu}_{0}+\boldsymbol{\Lambda}_{0} \boldsymbol{\eta}+\boldsymbol{\varepsilon}$ & Measurement model in Group $G=0$ \\
$\boldsymbol{\eta}=\boldsymbol{\alpha}_{0}+\mathbf{B}_{0} \boldsymbol{\eta}+\boldsymbol{\zeta}$ & Structural model in Group $G=0$ \\
\hline $\mathbf{y}=\boldsymbol{\nu}_{1}+\boldsymbol{\Lambda}_{1} \boldsymbol{\eta}+\boldsymbol{\varepsilon}$ & Measurement model in Group $G=1$ \\
$\boldsymbol{\eta}=\boldsymbol{\alpha}_{1}+\mathbf{B}_{1} \boldsymbol{\eta}+\boldsymbol{\zeta}$ & Structural model in Group $G=1$
\end{tabular}

where $G=1$ denotes the subgroup cover (or short: subgroup) and $G=0$ the subgroup complement (short: complement). The model is specified for the subgroup and the complement where parameters can either differ between the groups or be assigned with equality constraints (e.g., for invariant measurement models). For example, when comparing structural parameters between subgroup and complement (e.g., the means of a latent variable), measurement invariance for the latent construct should be assumed in order to appropriately compare the structural parameters (Meredith, 1993). However, if groups with measurement variance or differential item functioning from a psychometric test are under investigation, the measurement model parameters should not necessarily be set equal between groups.

The parameters of the two-group SEM can be estimated simultaneously with a sample of $N$ independent and identically distributed realizations of $\mathbf{y}$ using standard maximum-likelihood theory. Let $\boldsymbol{\theta}$ denote the vector of all parameters for all groups and assuming a conditional multivariate normal distribution of $\mathbf{y}$ for every group $G=g$, the log-likelihood function of a multigroup SEM (Browne \& Arminger, 1995; Jöreskog, 1971) is:

$$
\log L(\boldsymbol{\theta})=-\frac{1}{2} \sum_{g=0}^{1} n_{g}\left[\log \left|\boldsymbol{\Sigma}_{g}\right|+\operatorname{tr}\left(\boldsymbol{\Sigma}_{g}^{-1} \mathbf{H}_{g}\right)\right]+c
$$

where $\mathbf{H}_{g}=\mathbf{S}_{g}+\left(\overline{\boldsymbol{\nu}}_{g}-\boldsymbol{\mu}_{g}\right)\left(\overline{\boldsymbol{\nu}}_{g}-\boldsymbol{\mu}_{g}\right)^{\mathrm{T}}$, and for each of the groups, $\mathbf{S}$ is the sample variance-covariance matrix; $\boldsymbol{\Sigma}_{g}$ is the model-implied variance-covariance matrix; $\overline{\boldsymbol{\nu}}_{g}$ is the 
sample mean vector; $\boldsymbol{\mu}_{g}$ is the model-implied mean vector; $n_{g}$ is the group size, and $c$ is a constant. Two example specifications of such a two-group SEM are given in our illustrative examples below.

Notice that the SubgroupSEM approach is not necessarily limited to the specification and estimation procedure of a two-group structural equation model given above. The rationale presented in this paper also applies if, for example, a full information maximum likelihood (FIML; Molenberghs, Fitzmaurice, Kenward, Tsiatis, \& Verbeke, 2015 ) estimator is used to account for missing data or a multilevel extension of SEM (Lüdtke et al., 2008, Mehta \& Neale, 2005) is used (both of which we illustrate in our empirical examples later on). In this sense, the two-group SEM is chosen for explanatory reasons to illustrate the steps of subgroup discovery in structural equation models.

\section{Step 2: Choosing Covariates or: The Search Space}

After specifying our target concept, we choose a set of continuous and/or categorical covariates potentially forming the subgroups of interest. The resulting search space consists of a large set of subgroup descriptions (also called patterns) that can be formed from these covariates. Each subgroup description is composed of univariate selection expressions known as selectors (sometimes also named conditions or basic patterns). For example, if we consider age as a covariate, age $>20$ would be a selector, selecting all persons whose age is over 20 .

Subgroup descriptions are derived as combination of selectors through Boolean formulas (Lemmerich, 2014). For example, if we want to search for subgroups based on participants' age and gender, we can choose these variables as covariates. The subgroup discovery algorithm will then select different combinations of selectors, e.g. age $>20 \wedge$ gender $=$ diverse. Then, everyone fitting this subgroup description will be assigned $G=1$ (i.e., the subgroup) and everyone else will be assigned $G=0$ (i.e., the complement) and the two-group structural equation model from Step 1 will be estimated using this group variable $G$. Throughout the paper, we only consider conjunctive combinations. Disjunctive 
combinations are also possible, but far less common in exceptional model mining research.

In our implementation of SubgroupSEM, it is possible to include both categorical and continuous covariates to build the search space. For categorical covariates, each unique value is a selector. Continuous covariates can either be split automatically at reasonable intervals (e.g., quantiles) or manually divided into substantively meaningful categories beforehand. Some specialized subgroup discovery algorithms offer automatic online discretization, that is, the intervals of the continuous covariates are dynamically adapted to the subgroup discovery task at hand Carmona, Gonzalez, del Jesus, \& Herrera, 2010, del Jesus, Gonzalez, Herrera, \& Mesonero, 2007; Mampaey, Nijssen, Feelders, \& Knobbe, 2012).

\section{Step 3: Measuring Exceptionality or: The Interestingness Measure}

After the two-group SEM has been estimated for a subgroup and its complement, we proceed to measure how interesting the results for the particular subgroup are. The definition of "interestingness" must be aligned with individual research questions. That is, one defines an interestingness measure as a scoring function of the target concept (i.e., the two-group SEM) on the subgroup based on what is of interest in a specific research

context. Similar as proposed for the special case of latent growth curve modeling (Mayer et al., 2021), we suggest two different classes of interestingness measures for SubgroupSEM, namely, test-statistic based interestingness measures and exploratory interestingness measures:

The first group of interestingness measures allows for a principled, statistically oriented selection of subgroups. That is, the question whether a subgroup is interesting (or: exceptional) is determined by the test statistic of a test on the model parameters. In our setting, we can use statistics of a Wald test, likelihood ratio test, or score test to define an interestingness measure. For example, within each two-group SEM, we can compute a Wald test statistic with the null hypothesis that all means of the latent variables are the same in the subgroup and its complement. The test statistic increases as the equality of latent means becomes statistically less plausible. Notice that we explicitly suggest using a 
test statistic (e.g., a z-value from a Wald test) and not the statistical test itself (e.g., the corresponding $p$-value). A test statistic can be considered a function of the estimated parameters taking into account the amount of estimation uncertainty; that is, such an interestingness measure implicitly accounts for sample size and sampling variability. However, a statistical test requires further information on the asymptotic distribution of the test statistic and corrections for multiple testing, neither of which is easily available for subgroup discovery algorithms (a topic addressed by Hämäläinen \& Webb, 2019, which we also treat in Appendix B. In our software implementation, user-friendly functions for conducting subgroup discovery using the test statistic of a likelihood ratio test (comparing the two-group SEM to a baseline model) and a Wald test statistic with user-defined constraints are available. Statistical details of these procedures are given in Appendix A.

A second group of interestingness measures is rather exploratory in nature and is meant to be used in an interactive and iterative fashion. Here, we typically trade off the amount of deviation of subgroup parameters from the complement's parameters against the size of the subgroup (i.e., the number of individuals $n$ covered by the subgroup). Formally,

a distance measure $\Delta$ of model parameters (e.g., absolute difference in a parameter between subgroup and complement) is multiplied by a factor $n^{a}$, where $a \in[0,1]$ is a user-chosen parameter determining the weight of the factor (cf. Klösgen, 1996, Leman et al., 2008). For $a=0$ the subgroup size is not taken into account, and only the distance measure serves as an interestingness measure. With increasing $a$, the subgroup size is weighted more strongly. The distance measure $\Delta$ can also be derived from multiple parameters, for example, using a sum of elementwise squared differences. As stated before, interestingness measures which are derived this way allow for interactive and iterative exploration of potential subgroups and can be tailored to the research question at hand (Mayer et al., 2021).

\section{Step 4: Finding Subgroups or: The Search Strategy}

So far, we have described how the targeted structural equation model can be specified, how subgroup descriptions are derived from the covariate space, and how the 
interestingness of a particular subgroup can be measured. In the last step, we pass our model specification, covariates, and interestingness measure to a subgroup discovery algorithm, e.g., depth-first search or beam search. As a result, the algorithm returns the $k$ most interesting subgroups, their descriptions, their interestingness measure values, and the respective group size.

Subgroup discovery can employ a variety of potential search algorithms, starting from an exhaustive brute force method, more refined algorithms that are still exhaustive and efficient, and finally heuristics like beam search. In its simplest form, subgroup discovery estimates the model for each possible combination of the selectors and lists them ordered by their interestingness measure. However, this method quickly becomes computationally infeasible with an increasing number of covariates (and hence selectors) and as the target SEM becomes increasingly complex. More refined algorithms offer the possibility to bound the search space by giving an early indication of whether a subgroup will not (or is unlikely to) provide a (more) interesting result. In exceptional model mining, use of the beam search heuristic is common (cf. Duivesteijn et al., 2016) as it explores large covariate spaces efficiently, but at the cost of not necessarily arriving at an optimal solution (i.e., unvisited subgroups could yield higher values of the interestingness measure).

Thus, a key part of subgroup discovery is the availability of pruning strategies to reduce the magnitude of the search space. The algorithms have different possibilities to incorporate pruning strategies, for example, depending on their enumeration of subgroups (i.e., the order in which subgroups are visited) as this dictates which information is available for early detection of relevant subgroups. A distinction is drawn between safe pruning strategies, which guarantee that excluded subgroups are not relevant to the final result, and heuristic strategies (e.g., in beam search), for which this is only unlikely. In general, the derivation of a safe pruning strategy for a target SEM depends on the specific interestingness measure and can be mathematically complex. However, in high-stakes situations, the guarantee of arriving at an optimal solution might be worth the effort. 
The details of these algorithms and their components are given in the following section. As the search space grows exponentially with each covariate added, the search algorithm should be chosen according to the size of the search space and under consideration of available options (e.g., pruning through optimistic estimates) to trim the search space. This allows computational demands and search efficiency to be balanced. The full four-step procedure is illustrated with two real-data examples below.

\section{Subgroup Discovery Algorithms}

The complexity of a SubgroupSEM task is mainly driven by two aspects: the estimation procedure for the structural equation modeling and the magnitude of the search space. On the one hand, the estimation of a single model can be time-consuming, especially if multilevel structures or marginal likelihoods are involved. Hence, it is beneficial to select the details of the estimation procedure with care to speed up computations. On the other hand, even for a relatively small number of selectors, an exponentially large number of conjunctive patterns can be generated. Thus, a significant amount of research in the computer science literature has been dedicated to enabling efficient subgroup discovery even in larger search spaces. This can be achieved by applying pruning strategies and different search algorithms.

In the following, we present the technical details necessary to adapt the subgroup discovery task at hand to the respective complexity of the target SEM and the magnitude of the search space. However, we presume a familiarity with choosing efficient estimation procedures for SEM, for example, skipping estimation of standard errors if not needed or preferring least square estimators over marginal likelihoods if categorical variables are included. Consequently, we will focus on the subgroup discovery side of efficiently traversing the search space. See Lemmerich (2014) for a more detailed overview from a computer science perspective. 


\section{Pruning Strategies}

A key concept of efficient subgroup discovery is the selection of branches of subgroups that are not visited by the search algorithm, known as pruning. We distinguish between two types of pruning strategies: First, a general strategy is the specification of anti-monotone constraints, for example, constraining the minimum subgroup size $N_{\min }$ or the search depth $d$. These are called anti-monotone because if the constraint is not satisfied in a subgroup, then none of its specializations will satisfy the constraint. Hence, a whole branch of subgroups can be pruned. These strategies are already very helpful in restricting an otherwise exponentially growing search space. Second, a more specialized approach is to compute optimistic estimates (Grosskreutz, Rüping, \& Wrobel, 2008; Wrobel, 1997) or upper bounds for the interestingness measure of yet unvisited subgroups. For example, if we are looking for the $k$ best subgroups and can show that none of the unvisited subgroups can yield a better interestingness measure compared to the $k$ best already-visited subgroups, we can prune the search tree.

We illustrate the concept of optimistic estimates with a reduced form of our math test example (three schools and grades instead of five) in Table 3 . As a reminder, subgroups were formed based on two covariates, namely, the school variable $A$ and the grade variable $B$. We assume to search for the single best group, to have already computed the interestingess measure for single selector subgroups, to have computed an optimistic estimate for each of these subgroups. From these optimistic estimates, we can derive an upper bound for the interestingness measures of all specializations of the respective subgroup. For example, we may have found an interestingness measure of 2 for subgroup $A=1$ and derived an optimistic estimate of 4 for its three specializations (i.e., $A=1 \wedge B=1,2,3)$, which means none of the three specializations can yield an interestingness measure higher than 4. Before we even start to examine the two selector subgroups, we can already conclude that the specializations of $A=1, B=1$, and $B=2$ could not yield a better IM than already found (i.e., $>7$ ). Thus, the upper bounds allow 
SUBGROUPSEM

Table 3

Simplified illustrative example for utilization of optimistic estimates

\begin{tabular}{lll}
\hline Subgroup & $\begin{array}{l}\text { Interestingness } \\
\text { Measure }\end{array}$ & $\begin{array}{l}\text { Optimistic } \\
\text { Estimate }\end{array}$ \\
\hline$A=1$ & 2 & 4 \\
$A=2$ & 5 & 8 \\
$A=3$ & 7 & 9 \\
$B=1$ & 4 & 5 \\
$B=2$ & 5 & 6 \\
$B=3$ & 6 & 9
\end{tabular}

\begin{tabular}{ll}
\hline Specialization & Upper Bound \\
\hline$A=1 \wedge B=1$ & 4 \\
$A=1 \wedge B=2$ & 4 \\
$A=1 \wedge B=3$ & 4 \\
$A=2 \wedge B=1$ & 5 \\
$A=2 \wedge B=2$ & 6 \\
$A=2 \wedge B=3$ & 8 \\
$A=3 \wedge B=1$ & 5 \\
$A=3 \wedge B=2$ & 6 \\
$A=3 \wedge B=3$ & 9
\end{tabular}

Note. On the left side the IM for single selector groups are already computed along with an optimistic estimate, which is an upper bound for the IMs of the subgroups' specializations. The right side illustrates the upper bound for the IM of each specialization derived as the minimum of the two respective generalizations' optimistic estimates.

us to skip seven out of the nine specializations of the remaining search space (as illustrated on the right side of Table 3). We will see that some algorithms make it sufficient to inspect one out of the nine specializations under some circumstances, thereby reducing the search space drastically. This illustrates how efficient optimistic estimates can be for pruning.

Notable applications of applying optimistic estimates in exceptional model mining include, for example, Duivesteijn et al. (2012), who used Cook's distance to mine regression models and derived different bounds for Cook's distance. An overview over the derivation of several optimistic estimates (e.g., mean-based, variance-based) is given by Lemmerich, Atzmueller, and Puppe (2016). While we are not aware of prior derivation of optimistic estimates directly adaptable to SEM, we will discuss this as a promising field for future research later on. 


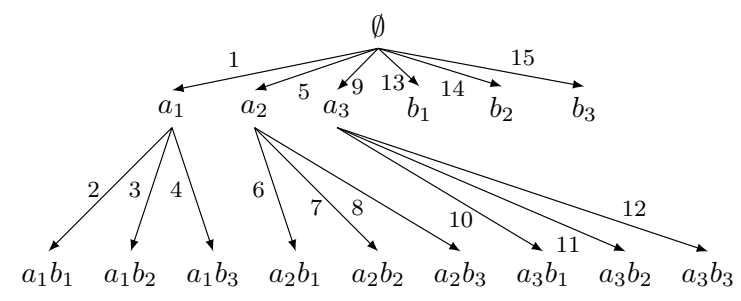

(a) Depth-first search

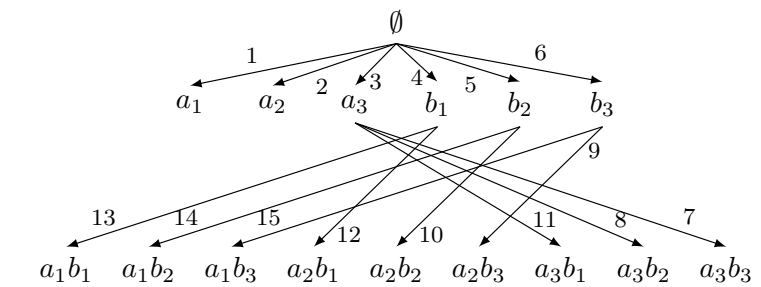

(c) Best-first search

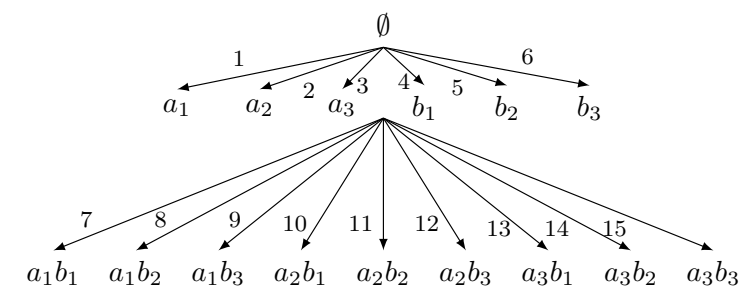

(b) Apriori algorithm

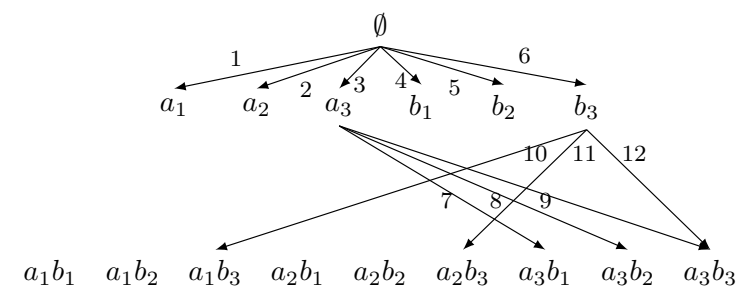

(d) Beam search

\section{Figure 3}

Illustration of subgroup discovery algorithm traversing the search space of our math test example and corresponding to the hypothetical values in Table 3 Numbered arrows indicate in which order the subgroups are examined by the algorithm.

\section{Algorithms}

In general, we distinguish between exhaustive algorithms, which guarantee an optimal solution, and heuristic algorithms that aim to find good but not necessarily optimal subgroups more quickly by focusing on the most promising parts of the search space. Note that exhaustive algorithms will arrive at the same (optimal) solution, but they will differ with regard to computational time and memory efficiency depending on the pruning strategy.

In this section, we present four established subgroup discovery algorithms, namely, depth-first search, an apriori algorithm (Kavšek, Lavrač, \& Jovanoski, 2003), best-first search (Webb, 1995), and, as a heuristic method, beam search (Clark \& Niblett, 1989). These differ with regard to (a) the order in which the subgroups are visited; (b) how well pruning can be applied; (c) how memory-intensive the algorithm is; (d) and whether they are exhaustive or heuristic. The algorithms are also illustrated in Figure 3 based on the reduced version of our math test example (again, three schools and grades instead of five). 


\section{Depth-first search}

One very popular and simple strategy for subgroup discovery is depth-first search. This algorithm starts with a subgroup described by one selector and deepens the search by iteratively adding selectors to the subgroup. As soon as the maximum search depth or any other pruning criterion is reached, the algorithm returns to a higher level of the search tree and explores the remaining specializations of the subgroup in the same manner (Lemmerich, 2014).

In our example, this algorithm would start by estimating the model for school $A=1$, followed by the grades within this school (i.e., $A=1 \wedge B=1, \ldots$ ), then proceeding with school $A=2$ and its grades, and so on. This is illustrated in the upper left panel of Figure 3. A so-called optimal refinement operator ensures that each subgroup is only visited once (which is not the case for all algorithms). While depth-first search is very memory efficient, it has a limited capability to integrate pruning through optimistic estimates. For example, most information typically required for deriving optimistic estimates is only available if most of the subgroups have already been visited.

\section{Apriori algorithm}

The Apriori algorithm is a levelwise approach to subgroup discovery. It starts by visiting all subgroups described by a single selector first and then continues with iteratively evaluating two selector subgroups, three selector subgroups, and so on. The main advantage of an Apriori algorithm is that it facilitates pruning, because all corresponding generalizations of a subgroup under evaluation were already evaluated (Lemmerich, 2014).

For our example, the Apriori algorithm is illustrated in the upper right panel of Figure 3. The algorithm starts by estimating the model for each school $(A=1,2,3)$ and each grade $(B=1,2,3)$ and then proceeds with all combinations of the two variables. If the pruning technique illustrated in Table 3 was applied, only the specializations $a_{2} b_{3}$ and $a_{3} b_{3}$ would be inspected and the estimation of models $a_{1} b_{1}, a_{1} b_{2}, a_{1} b_{3}, a_{2} b_{1}, a_{2} b_{2}, a_{3} b_{1}$, and $a_{3} b_{2}$ could be omitted due to the available information from the first level. Thus, a 
substantial reduction of the search space can be achieved. However, this would come at the cost of increased memory requirements, because the information from previous levels has to be stored during the search.

\section{Best-first search}

Best-first search also starts by visiting all subgroups described by a single selector. For each evaluated subgroup, all specializations are inserted into a list of open candidates, which is sorted by an upper bound derived from optimistic estimates. The algorithm continues with evaluating the current top subgroup of this list, removing it from the list and inserting all its specializations that cannot be pruned. The search stops once the list is empty. Similar to a levelwise approach, this algorithm ensures taht the generalizations of a subgroup under evaluation were already evaluated, which supports pruning (Lemmerich, 2014).

The different ordering of the investigated patterns compared to the Apriori algorithm plays an important role, as can be seen in the lower left panel of Figure 3. While the investigation of the first level as well as the pruning procedure is identical to Apriori, best-first search proceeds by exploring the specializations of the most promising node. In our example, that would be school $A=3 \wedge B=3$. If this subgroup would indeed yield an $\mathrm{IM} \geq 8$ (i.e. better than already found), the subgroup discovery could stop here after examining only one of the possible specializations, because none of the remaining specializations could lead to a higher IM. Thus, best-first search is optimal for exploiting optimistic estimates.

\section{Beam search}

Beam search is a heuristic search strategy based on the intuition that interesting subgroups also are more likely to have interesting specializations. During the search, the currently best $w$ subgroups are stored in a list (the "beam") of length $w$, the beam width (Lemmerich, 2014). This is probably the most popular algorithm for heuristic subgroup discovery and exceptional model mining, with several improvements suggested in the last 
decade. These include the DSSD (diverse subgroup set discovery) algorithm (van Leeuwen $\&$ Knobbe, 2012), which seeks to reduce redundancy within the $k$-top subgroups, and double beam search (Valmarska, Lavrač, Fürnkranz, \& Robnik-Šikonja, 2017), which allows for different interestingness measures for rule refinement and rule selection.

Beam search starts by visiting all subgroups described by a single selector and stores the best $\mathrm{w}$ subgroups within the beam. Then, all specializations of the subgroups within the beam are evaluated. If a more interesting subgroup is found, it is stored in the beam in exchange for a less interesting subgroup. This procedure is iteratively continued until no better subgroup than those already in the beam can be found (Lemmerich, 2014). This is illustrated in the lower right panel of Figure 3 with a beam width of 2 . Note that beam search uses a full refinement operator; that is, it is possible for a subgroup to be visited more than once if it is a specialization of multiple candidates in the beam (e.g., the subgroup $a_{3} b_{3}$, which corresponds to $A=3 \wedge B=3$ in Figure 3). Conversely, not every subgroup is visited by the algorithm, as is the case for $a_{1} b_{1}, a_{1} b_{2}, a_{2} b_{1}$, and $a_{2} b_{2}$ in our reduced math test example. The beam width can help to effectively reduce the search space without needing to calculate optimistic estimates. However, this comes at the cost of not necessarily arriving at an optimal solution.

Duivesteijn et al. (2016) offer a detailed introduction to beam search for exceptional model mining, including an explanation of a pseudo-code example as well as a comprehensive runtime analysis depending on search depth, beam width, model complexity.

\section{Table 4}

Summary table for subgroup discovery algorithms

\begin{tabular}{lllll}
\hline & DFS & Apriori & BFS & Beam \\
\hline exhaustive search & yes & yes & yes & no \\
memory efficient & yes & no & no & yes \\
optimistic estimates effect & medium & high & high & low \\
\hline
\end{tabular}

Note. DFS = depth-first search; BFS = best-first search; exhaustive search distinguishes whether the covariate is searched exhaustively or heuristically; memory efficient distinguishes whether the algorithm makes efficient use of memory or is very memory demanding; optimistic estimates effect distinguishes how well optimistic estimates can be utilized by the algorithm. 


\section{SubgroupSEM Package}

Along with this paper, we provide an open source implementation of the SubgroupSEM approach within the statistical programming language $\mathrm{R}$ ( $\mathrm{R}$ Core Team, 2021). Our package subgroupsem provides an interface between the Python module pysubgroup (Lemmerich \& Becker, 2019) and the structural equation modeling R package lavaan (Rosseel, 2012). While pysubgroup implements state-of-the-art subgroup discovery algorithms, including depth-first search, Apriori, best-first search, and beam search, the lavaan package is very popular for SEM in $\mathrm{R}$ amd enables the estimation of multigroup models, multilevel models, full information maximum likelihood, and robust estimation procedures.

Our subgroupsem package implements SEM as the target model for subgroup discovery and uses the reticulate package (Ushey, Allaire, \& Tang, 2021) for interfacing between Python and R. We provide user-friendly methods for conducting subgroup discovery using user-defined interestingess measures, or, for example, interestingness measures derived from a likelihood ratio test or a Wald test with user-defined constraints. Listing 1 gives an overview of how the four steps of SubgroupSEM can be carried out with our R package. Details on the installation, the complete code for all examples in this paper, as well as the package versions of subgroupsem and pysubgroup can be obtained from the online supplementary material. Here, we follow the suggestions of Peikert and Brandmaier (2021) and also provide Dockerfiles to ensure sustainable reproducibility of our code and results.

\section{Illustrative Examples}

In this section, we apply the SubgroupSEM approach to two empirical examples. We will start off with an examination of measurement invariance in a confirmatory factor analysis. In the second example, we identify exceptional mediation patterns in a mediated latent growth curve model using data from a large-scale assessment study. 


\section{Example 1: Differential Item Functioning in Confirmatory Factor Analysis}

In our first example, we re-analyse the popular mental ability dataset by Holzinger and Swineford (1939). It contains scores for nine mental ability tests measuring visual, textual, and speed ability for $N=301$ students and is freely available via, for example, the lavaan package in $\mathrm{R}$ (Rosseel, 2012). This particular example analysis was also used by Serang et al. (2021), which makes it an adequate example to study the similarities and differences between the decision tree approach and the SubgroupSEM approach.

\section{Model Specification}

In the Holzinger \& Swineford dataset nine scores from mental ability tests are linked to three latent variables, namely: visual ability $\eta_{\text {visual }}$ measured with visual perception $X_{1}$, cubes $X_{2}$, and lozenges $X_{3}$; textual ability $\eta_{\text {textual }}$ measured with paragraph comprehension $X_{4}$, sentence completion $X_{5}$, and word meaning $X_{6}$; and speed ability $\eta_{\text {speed }}$ measured with speeded addition $X_{7}$, speeded counting of dots $X_{8}$, and speeded discrimination of straight and curved capitals $X_{9}$. The corresponding confirmatory factor analysis model is shown in Figure 4. After loading the required packages in R, the model can be specified using lavaan syntax as follows:

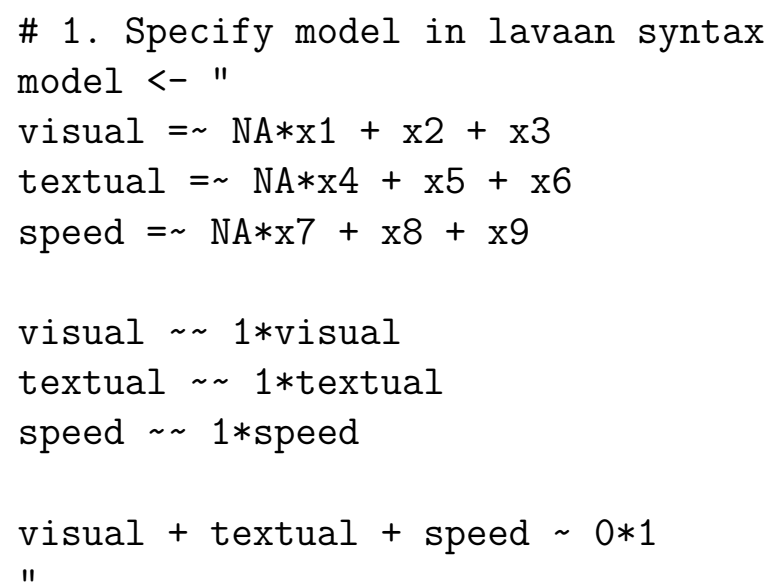

For a comprehensive tutorial on the lavaan syntax, refer to Rosseel (2012). Here, we specify that the latent variables are measured by the respective indicators and fix the 
latent variances to 1 and the latent means to 0 for identification.

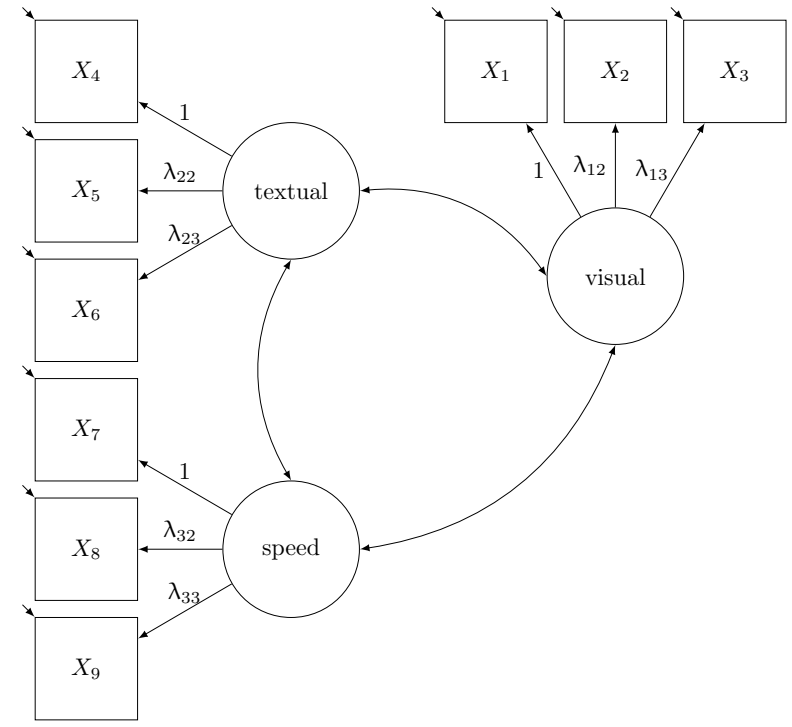

Figure 4

Path diagram of the three factor model for the Holzinger \& Swineford data used in Example 1.

\section{Covariates}

In a second step, we specified the search space by choosing the relevant covariates to form subgroup descriptions. In this example, we included three available covariates, namely sex (with levels "female" and "male"), grade (with levels 7 and 8), and school (with levels "Pasteur" and "Grant-White"), to keep our results comparable to the computations by Serang et al. (2021). In subgroupsem, this selection is conducted via a vector of variable names:

\# 2. Choose covariates

covariates <- c("sex", "school", "grade")

\section{Interestingness Measure}

In the third step, an interestingness measure $\mathrm{IM}_{1}$ was defined. As we were not interested in differences on single parameters, but potential measurement invariance as a whole, we applied a likelihood ratio test statistic as interestingness measure. That is, the 
proportional increase in the log-likelihood of the two-group structural equation model with subgroup and complement compared to a single-group baseline model was computed (cf. Appendix A. Thus, subgroups are selected such that identifying heterogeneous groups will significantly improve the model fit.

In subgroupsem, the LRT-based interestingness measure is implemented as its own wrapper function, so no further specifications are necessary other than running:

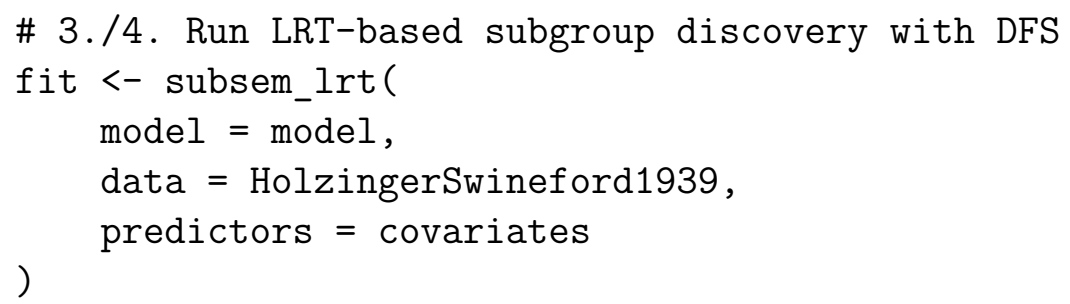

This will by default use a simple depth-first-search algorithm, which is a reasonable choice given the rather small search space. Note that the subsem_lrt function uses the likelihood test statistic itself and does not apply significance testing to this value. This is advantageous because we do not have to assume a $\chi^{2}$ distribution of the test statistic and avoid the problem of multiple testing in the subgroup search.

While the above interestingness measure based on the LRT statistic would be expected to provide similar results as the approach by Serang et al. (2021), who also used an LRT-based criterion, we defined a second, exploratory interestingness measure to illustrate how such a measure can be used in an interactive way. The second interestingness measure $\mathrm{IM}_{2}$ was defined as

$$
\mathrm{IM}_{2}=\sqrt{N_{s g}} \cdot \sum_{j=1}^{3}\left|\lambda_{0 j}-\lambda_{1 j}\right|
$$

which is the sum of absolute differences in factor loadings between the subgroup and its complement for the latent variable visual ability weighted by the square root of the subgroup size $N_{s g}$. 
We can calculate this interestingness measure via lavaan by adding some changes to our initial model syntax:

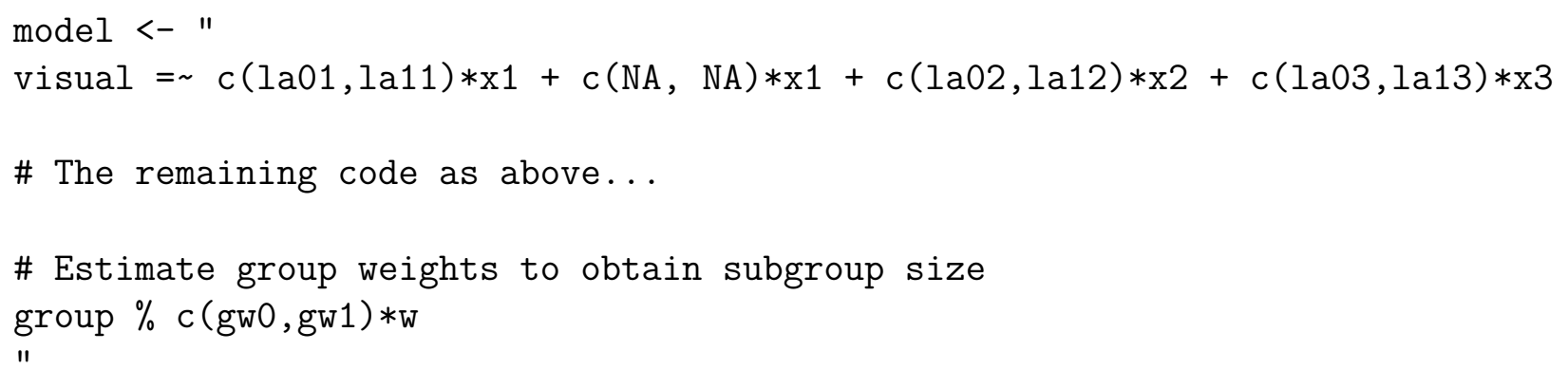

so that we have labels for the loadings and additionally obtain a group weight parameter. Then, we can calculate our interestingness measure as a user-defined parameter using the := operator. The subsem_qf parameter will automatically be detected as the interestingness measure:

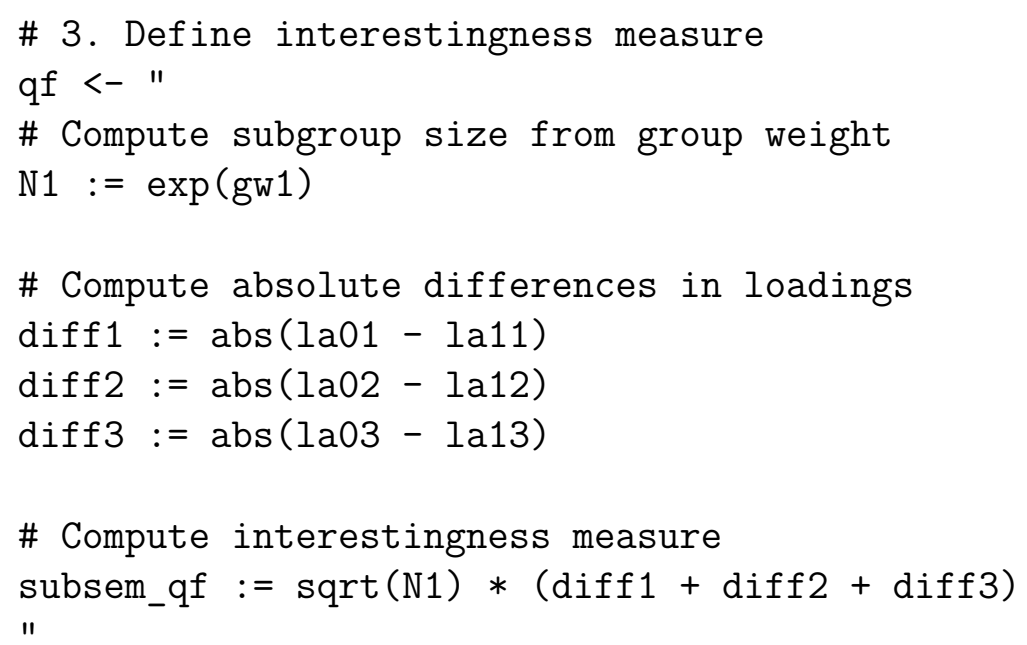

Finally, we can pass our model syntax, dataset, covariate selection, and interestingness measure to the subsem wrapper function:

\# 4. Run subgroup discovery with DFS

fit $<-$ subsem ( 


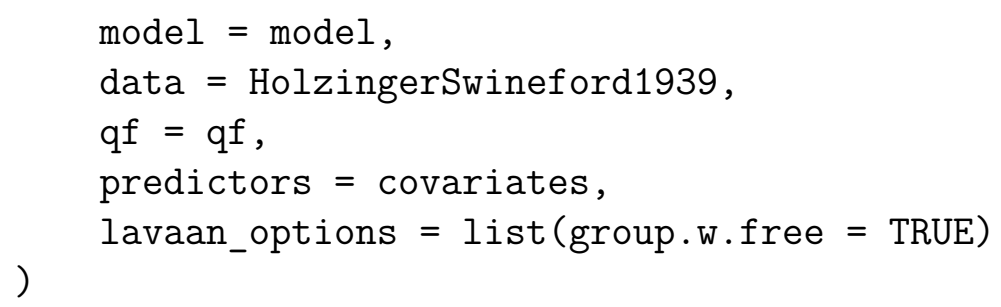

Note that estimation of the group weight, which is necessary to obtain the subgroup size as model parameter, has to be enabled via lavaan_options. Again, in this example a simple depth-first search is run by default.

\section{Results}

We conducted the subgroup discovery task with a maximum search depth $d=3$ (which is the maximum possible depth in this case): that is, a subgroup description could contain a selector from each covariate. The analysis did have a lower bound of $N_{\min }=30$ for subgroup size, which is default in subgroupsem.

We can obtain the results of our subgroup discovery task using the generic summary() function (here, for our first interestingness measure):

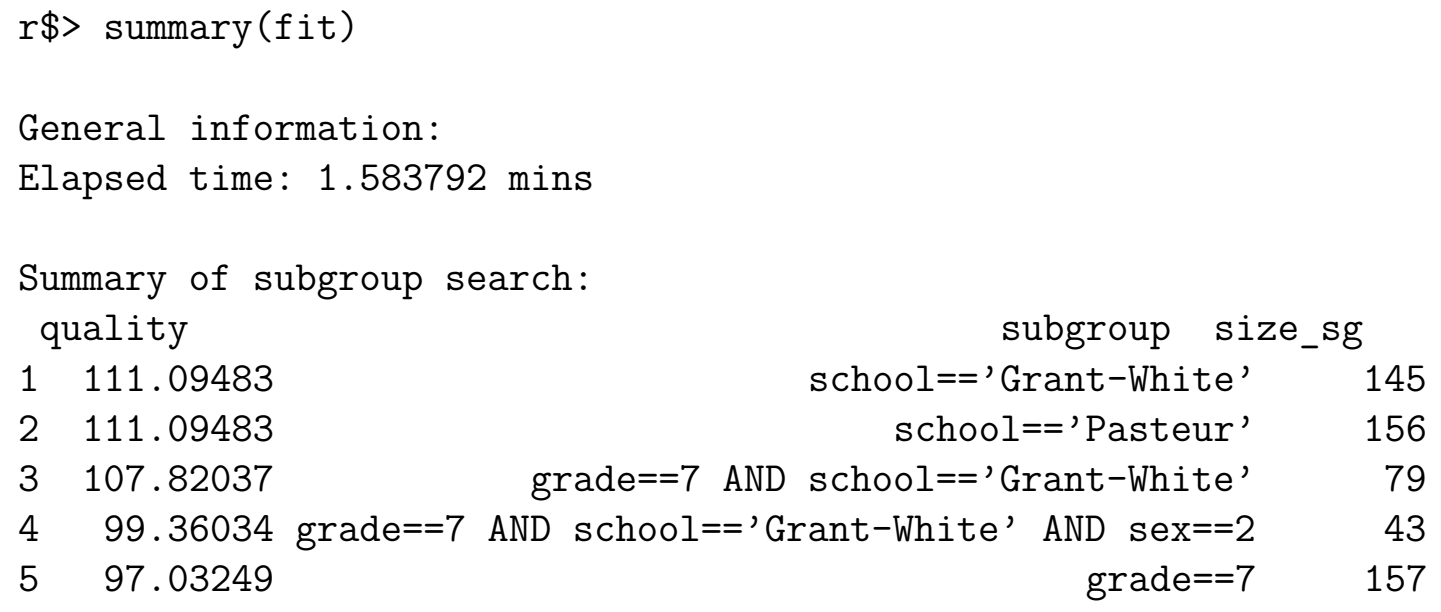

The five most interesting subgroups and their description, size, and corresponding interestingness score (i.e., the likelihood ratio test statistic LR) were returned. The results for both interestingness measures are summarized in Table 5 . The results on the left side of the table correspond to the above $\mathrm{R}$ output. 


\section{Table 5}

Description of subgroups in confirmatory factor analysis example

\begin{tabular}{|c|c|c|c|c|c|c|c|c|c|}
\hline & T-based inte & stingness mea & re II & & & erences-base & terestingne: & nea & Ire $\mathrm{IM}_{2}$ \\
\hline \# & $\begin{array}{l}\text { Subgroup } \\
\text { description }\end{array}$ & Value & Size & Measure & \# & $\begin{array}{l}\text { Subgroup } \\
\text { description }\end{array}$ & Value & Size & Measure \\
\hline 1 & School & Pasteur & 156 & 111.095 & 1 & $\begin{array}{l}\text { School } \\
\text { Sex }\end{array}$ & $\begin{array}{l}\text { Pasteur } \\
\text { Male }\end{array}$ & 82 & 7.025 \\
\hline 2 & School & Grant-White & 145 & 111.095 & 2 & School & Pasteur & 156 & 6.890 \\
\hline 3 & $\begin{array}{l}\text { School } \\
\text { Grade }\end{array}$ & $\begin{array}{l}\text { Grant-White } \\
7\end{array}$ & 79 & 107.820 & 3 & School & Grant-White & 145 & 6.643 \\
\hline 4 & $\begin{array}{l}\text { School } \\
\text { Sex } \\
\text { Grade }\end{array}$ & $\begin{array}{l}\text { Grant-White } \\
\text { Male } \\
7\end{array}$ & 43 & 99.360 & 4 & Sex & Male & 155 & 5.533 \\
\hline 5 & Grade & 7 & 157 & 97.032 & 5 & Sex & Female & 146 & 5.370 \\
\hline
\end{tabular}

Note. The likelihood ratio test statistic LR) is used as interestingness measure on the left side and the weighted absolute differences for factor loadings of visual ability on the right side.

The first subgroup consisted of students from the Pasteur school and contained $N=156$. The second subgroup consisted of students from the Grant-White school, which is the complement to the first subgroup (containing $N=145$ students) and resulting in the same interestingness measure of $L R=111.095$. The third subgroup consisted of 7 th grade students from Grant-White school, which is a specialization of the second subgroup (containing $N=79$ students) with an interestingness measure of $\mathrm{LR}=107.820$. The fourth subgroup consisted of 7th grade male students from Grant-White school, which is a specialization of the second and third subgroups (containing $N=43$ students) with an interestingness measure of $\mathrm{LR}=99.360$. The fifth subgroup consisted of 7 th grade students, which is a generalization of the third subgroup (containing $N=157$ students) with an interestingness measure of $L R=97.032$. The factor loadings and intercepts for the confirmatory factor analysis within each subgroups are given in Table 6 and compared to the factor loadings of the baseline model.

Our results are similar to those by Serang et al. (2021), who applied the SEM tree approach to this example dataset. In their analysis, the algorithm chose to make only a single split, namely by school, which are also the most interesting subgroups in our analysis. However, the SubgroupSEM approach returned further specializations and partly 
Table 6

Factor loadings and intercepts in confirmatory factor analysis of subgroups compared to the baseline model for LRT-based interestingness measure

\begin{tabular}{|c|c|c|c|c|c|c|}
\hline Parameter & Baseline & Subgroup 1 & Subgroup 2 & Subgroup 3 & Subgroup 4 & Subgroup 5 \\
\hline$\nu_{1}$ & 4.936 & 4.930 & 4.941 & 4.772 & 4.810 & 4.746 \\
\hline$\nu_{2}$ & 6.088 & 6.200 & 5.984 & 6.054 & 6.070 & 5.932 \\
\hline$\nu_{3}$ & 2.250 & 1.996 & 2.487 & 1.862 & 1.805 & 2.097 \\
\hline$\nu_{4}$ & 3.061 & 3.317 & 2.823 & 3.148 & 3.372 & 2.828 \\
\hline$\nu_{5}$ & 4.341 & 4.712 & 3.995 & 4.446 & 4.605 & 4.123 \\
\hline$\nu_{6}$ & 2.186 & 2.469 & 1.922 & 2.329 & 2.399 & 2.020 \\
\hline$\nu_{7}$ & 4.186 & 3.921 & 4.432 & 3.542 & 3.534 & 3.823 \\
\hline$\nu_{8}$ & 5.527 & 5.488 & 5.563 & 5.223 & 5.167 & 5.238 \\
\hline$\nu_{9}$ & 5.374 & 5.327 & 5.418 & 5.057 & 5.121 & 5.166 \\
\hline$\lambda_{1}$ & 0.900 & 0.777 & 1.047 & 0.887 & 0.954 & 0.909 \\
\hline$\lambda_{2}$ & 0.498 & 0.572 & 0.412 & 0.576 & 0.725 & 0.462 \\
\hline$\lambda_{3}$ & 0.656 & 0.719 & 0.597 & 0.636 & 0.683 & 0.662 \\
\hline$\lambda_{4}$ & 0.990 & 0.971 & 0.946 & 0.964 & 1.137 & 0.943 \\
\hline$\lambda_{5}$ & 1.102 & 0.961 & 1.119 & 1.075 & 1.189 & 1.078 \\
\hline$\lambda_{6}$ & 0.917 & 0.935 & 0.827 & 1.010 & 1.075 & 0.900 \\
\hline$\lambda_{7}$ & 0.619 & 0.679 & 0.591 & 0.362 & 0.462 & 0.448 \\
\hline$\lambda_{8}$ & 0.731 & 0.833 & 0.665 & 0.592 & 0.617 & 0.513 \\
\hline$\lambda_{9}$ & 0.670 & 0.719 & 0.545 & 0.992 & 1.074 & 0.855 \\
\hline
\end{tabular}

overlapping subgroups (i.e., Subgroup 5 of 7 th grade students from both schools) that also exhibited exceptional measurement properties.

Furthermore, our additional analysis with another interestingness measure illustrates the flexibility of the SubgroupSEM approach in two ways: First, the interestingness measure can be tailored to a specific aspect of interest (e.g., loadings of a specific factor) with varying degrees of weighting for the subgroup size. This can be used interactively to explore measurement properties in certain subsets of the sample. Second, weighting for sample size introduces valuable information into the interestingness measure. While for the LRT-based interestingness measure a subgroup and its complement yield the same interestingness, the second IM presents the larger of these two groups as more interesting. 


\section{Example 2: Indirect and direct effects on growth curve trajectories}

In our second example, we apply subgroup discovery to a theory-based partially mediated latent growth model with multiple indicators and mediators proposed by DeVries, Szardenings, Doebler, and Gebhardt (2021). DeVries and colleagues examined whether and how the effects of special educational needs, socioeconomic status, reasoning ability, gender and school track on the trajectory of children's math competencies from Grade 5 to Grade 9 are mediated by children's global self-esteem and math-specific self-concept. They found that the theorized complex relationship among the variables provided a good fit to the data, along with significant mediating effects of global self-esteem and math-specific self-concept. However, contrary to the authors' predictions, the mediation effects were only observed for the baseline level of math competences in 5th grade (i.e., intercept of the growth curve), not for the actual growth over time (i.e., slope of the growth curve).

While the findings of DeVries et al. were partly contrary to their expectations, a further starting point may be to ask: "Is the hypothesized relation only true for a particular subset of students?" and generate hypotheses for future research. Here, the SubgroupSEM approach can help to identify subgroups for which the mediation process is exceptionally different from the rest of the sample in an exploratory fashion.

The original analysis was based on data from the National Educational Panel Study (Blossfeld, von Maurice, Bayer, \& Skopek, 2016), a multicohort large-scale longitudinal study that was administered for most cohorts until recently in yearly waves. Data from Starting Cohort Grade 5 (SC3), which was first sampled in fifth grade in late 2010 and 2011, was used, with focus on Grades 5, 7 and 9. Further information on variable selection and filter criteria can be found in DeVries et al. (2021) and in our supplementary material. In our SubgroupSEM analysis, we included data from $N=5753$ children and used full information maximum likelihood (FIML) estimation (in contrast to DeVries et al. who used multiple imputation) as it is less computationally demanding compared to estimating the SEM multiple times for each subgroup. In addition, we reproduced the original analysis 
finding close parameter estimates using FIML (which is in line with previous research comparing multiple imputation and FIML approaches, e.g., Collins, Schafer, \& Kam, 2001; Lee \& Shi, 2021).

\section{Model Specification}

The model by DeVries et al. consisted of three main parts: a measurement model for socioeconomic status, a latent linear growth curve model for math competencies in Grades 5,7 , and 9, and a mediation model with the intercept and slope of the growth curve as outcome variables. The complete model is displayed in the above left panel of Figure 5 .

Socioeconomic status was measured with four items asking the students whether they had "a desk to study" $\left(\mathrm{SES}_{1}\right)$, "learning software" $\left(\mathrm{SES}_{2}\right)$, "books that are useful for homework" $\left(\mathrm{SES}_{3}\right)$, and "a dictionary" $\left(\mathrm{SES}_{4}\right)$ at home. In our analysis, some parameters of the measurement model (i.e., intercepts and factor loadings) were constrained to be equal between subgroup and complement, while measurement error variances as well as the mean and variance of socioeconomic status could vary between groups.

The trajectory of math competencies from Grade 5 through Grade 7 to Grade 9 was modeled using a latent linear growth curve model, that is, a latent intercept and latent slope variable were specified. The measurement error variances in the math competency variables as well as the means, variances, and covariance of intercept and slope could differ between groups.

Finally, the multiple mediation model contained the predictor variables socioeconomic status, special educational needs, sex, reasoning ability, and school track; two mediator variables global self-esteem and math-specific self-concept; and the latent intercept and slope as outcome variables. All possible indirect and direct effects were included in the model. Additionally, we included the students' class ID to adjust the standard errors for (class-)clustered sampling (as the original authors did). 


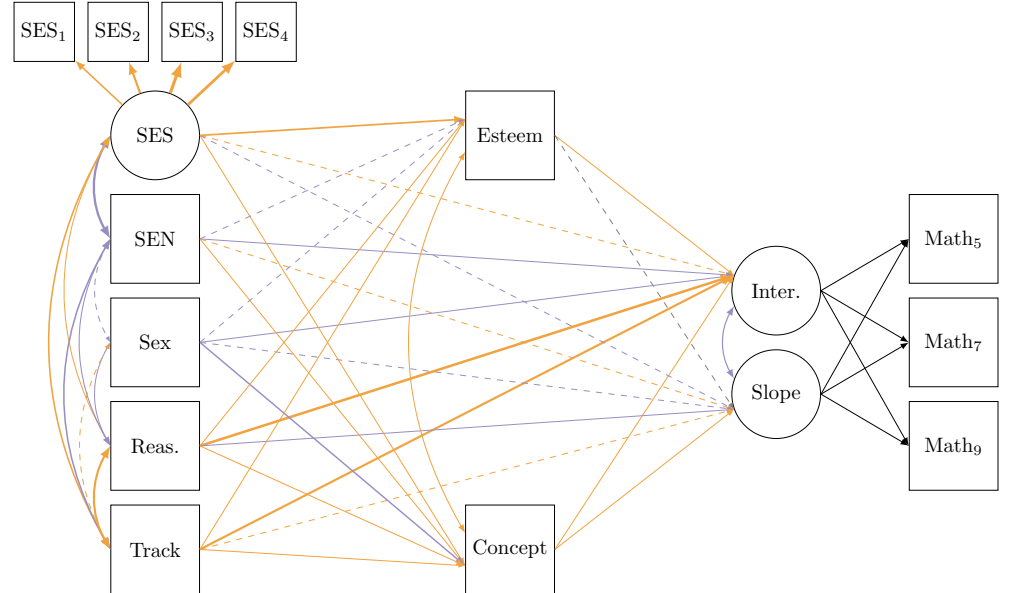

(a) Whole sample, $N=5753$

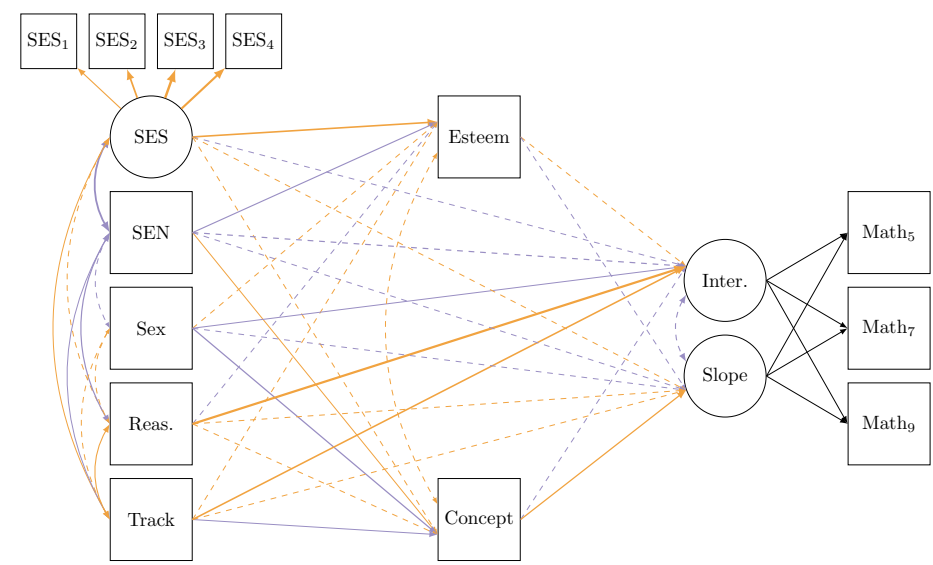

(c) Subgroup 2, $N=621$

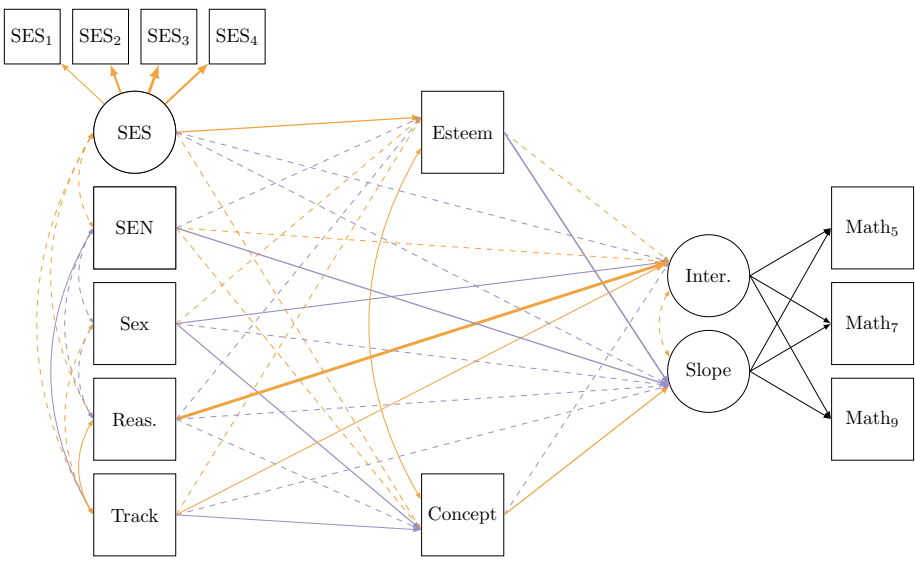

(b) Subgroup 1, $N=588$

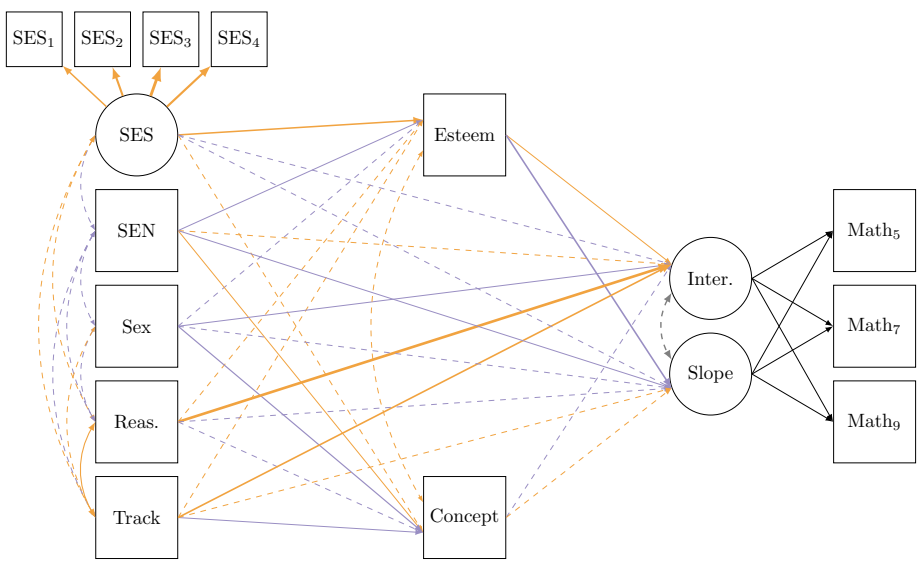

(d) Subgroup 3, $N=692$

\section{Figure 5}

Path diagram of the mediation and linear latent growth curve model by DeVries et al. (2021) for the NEPS data used in Example 2. Dashed lines indicate non-significant paths that were included in the model. Orange lines indicate a significant positive parameter estimate for the respective path, and purple lines indicate a significant negative effect. Thickness of the lines indicates the size of the standardized estimates. 


\section{Covariates}

The NEPS dataset contains a vast number of potential covariates, providing many opportunities for defining search spaces for subgroups. In this application, we want to leverage the power of beam search to explore this large search space. Thus, instead of selecting a manageable number of covariates based on our domain knowledge, our search space constitutes all variables containing valuable information about pupils at the beginning of the study. From the NEPS dataset based on questionnaires completed by the children themselves, we selected variables containing information collected at the beginning of Grade 5; with less than 20 percent missing values; that were not confidential and/or qualitative (i.e., open-ended answer format); and were not items of scales used in the model (i.e., global self-esteem and math-specific self-concept). This yielded a set of 154 variables spanning our search space, including idealistic and realistic educational aspirations, sociodemographic information about parents, satisfaction with several aspects of life, and parental support, to name just a few.

\section{Interestingness Measure}

The original authors' goal was to examine the mediation and indirect effects of students' global self-esteem and math-specific self-concept. To focus more deeply on this goal, we applied an interestingness measure to detect subgroups with exceptional patterns of indirect effects. More specifically, we specified a Wald test over all twenty indirect effects from the original model. Then, with regard to the two-group SEM from SubgroupSEM (i.e., comparing subgroup and complement), the Wald test statistic measures to what degree the indirect effects in a particular subgroup differ from the rest of the sample. Thus, subgroups with an overall diverging pattern of indirect effects can be found.

\section{Algorithm}

We conducted the subgroup discovery task using the beam search algorithm with a beam width of $w=50$. The maximum search depth was $d=2$; that is, a subgroup description could contain a selector of up to two covariates. The minimum required sample 
size was $N_{\min }=575$, which is roughly a tenth of the overall sample size. The algorithm should return the $k=3$ best subgroups. The $\mathrm{R}$ code for this example analysis is provided in the supplementary materials.

\section{Results}

The three most interesting subgroups and their description, size, and corresponding interestingness measure (i.e., the Wald test statistic) were returned and are summarized in Table 7. Figure 5 gives path diagrams indicating the size (i.e., line width corresponds to standardized parameter size), significance (i.e., straight lines for significant paths and dashed for non-significant) and direction (orange for positive effects, purple for negative effects) of each path in the complete sample and the three best subgroups. We refrain from discussing these paths in detail, as this would be beyond the scope of this illustration.

Table 7

Description of subgroups in mediated growth curve example

\begin{tabular}{|c|c|c|c|c|}
\hline$\#$ & Subgroup description & Value & Size & Measure \\
\hline 1 & $\begin{array}{l}\text { What grade did you have on last } \\
\text { year's final report card in math- } \\
\text { ematics? }\end{array}$ & $\begin{array}{l}\text { school-leaving certificate from a } \\
\text { middle-track school (Realschule / } \\
\text { Mittlere Reife) } \\
\text { satisfactory (3) }\end{array}$ & 588 & 170.307 \\
\hline 2 & $\begin{array}{l}\text { Verbal self-concept: I get good } \\
\text { grades in German. } \\
\text { What grade did you have on last } \\
\text { year's final report card in math- } \\
\text { ematics? }\end{array}$ & $\begin{array}{l}\text { somewhat applies } \\
\text { satisfactory (3) }\end{array}$ & 621 & 150.955 \\
\hline 3 & $\begin{array}{l}\text { Satisfaction with health } \\
\text { What grade did you have on last } \\
\text { year's final report card in math- } \\
\text { ematics? }\end{array}$ & $\begin{array}{l}\text { completely satisfied } \\
\text { satisfactory (3) }\end{array}$ & 692 & 148.118 \\
\hline
\end{tabular}

The first subgroup consisted of $N=588$ students with a realistic educational aspiration of graduating with a school-leaving certificate from a Realschule (i.e., a middle-track secondary school) and had a final math grade of satisfactory (i.e., 3 on a scale 


\section{Table 8}

Indirect effects in mediated latent growth curve model

\begin{tabular}{lrrrr}
\hline Indirect Effects & Full sample & Subgroup 1 & Subgroup 2 & Subgroup 3 \\
\hline $\mathrm{SES} \rightarrow$ Esteem $\rightarrow$ Intercept & $0.244^{*}$ & 0.182 & 0.260 & $0.5066^{*}$ \\
$\mathrm{SEN} \rightarrow$ Esteem $\rightarrow$ Intercept & -0.010 & -0.017 & -0.031 & $-0.060 *$ \\
$\mathrm{Sex} \rightarrow$ Esteem $\rightarrow$ Intercept & -0.001 & 0.004 & 0.001 & -0.002 \\
Reas. $\rightarrow$ Esteem $\rightarrow$ Intercept & $0.001 *$ & -0.001 & -0.001 & 0.001 \\
Track $\rightarrow$ Esteem $\rightarrow$ Intercept & $0.007 *$ & 0.001 & 0.001 & 0.012 \\
$\mathrm{SES} \rightarrow$ Concept $\rightarrow$ Intercept & $0.377^{*}$ & -0.024 & -0.039 & -0.073 \\
$\mathrm{SEN} \rightarrow$ Concept $\rightarrow$ Intercept & $0.072^{*}$ & -0.016 & -0.042 & -0.047 \\
Sex $\rightarrow$ Concept $\rightarrow$ Intercept & $-0.078 *$ & 0.011 & 0.028 & 0.019 \\
Reas. $\rightarrow$ Concept $\rightarrow$ Intercept & $0.010 *$ & 0.000 & 0.000 & 0.000 \\
Track $\rightarrow$ Concept $\rightarrow$ Intercept & $0.015 *$ & 0.017 & 0.0260 & 0.016 \\
SES $\rightarrow$ Esteem $\rightarrow$ Slope & -0.073 & -0.335 & -0.411 & $-0.511 *$ \\
SEN $\rightarrow$ Esteem $\rightarrow$ Slope & 0.003 & 0.032 & 0.049 & $0.061 *$ \\
Sex $\rightarrow$ Esteem $\rightarrow$ Slope & 0.000 & -0.007 & -0.001 & 0.002 \\
Reas. $\rightarrow$ Esteem $\rightarrow$ Slope & 0.000 & 0.001 & 0.001 & -0.001 \\
Track $\rightarrow$ Esteem $\rightarrow$ Slope & -0.002 & -0.002 & -0.002 & -0.012 \\
SES $\rightarrow$ Concept $\rightarrow$ Slope & $0.047 *$ & 0.045 & 0.040 & 0.045 \\
SEN $\rightarrow$ Concept $\rightarrow$ Slope & $0.009 *$ & 0.031 & 0.044 & 0.029 \\
Sex $\rightarrow$ Concept $\rightarrow$ Slope & $-0.010 *$ & $-0.021 *$ & $-0.030 *$ & -0.012 \\
Reas. $\rightarrow$ Concept $\rightarrow$ Slope & $0.001 *$ & 0.000 & 0.000 & 0.000 \\
Track $\rightarrow$ Concept $\rightarrow$ Slope & 0.002 & -0.033 & $-0.027 *$ & -0.010 \\
\hline
\end{tabular}

Note. SES = socioeconomic status; SEN = special educational needs; Intercept and slope refer to latent intercept and slope of linear growth curve model; concept = math-specific self-concept; esteem = global self-esteem

from 1 to 6 ) on their final report card last year (i.e., the grade before the survey). The interestingness measure (i.e., a Wald test statistic over the 20 indirect effects) had a value of $\mathrm{IM}=170.307$. As can be seen in Table 8, only one out of 20 indirect effects was significant. There was a negative indirect effect of sex on the slope of math compentency development mediated by math-specific self-concept.

The second subgroup consisted of $N=621$ students with a good verbal self-concept (i.e., the statement I get good grades in German somehat applies) and who had a final math grade of satisfactory (i.e., 3 on a scale from 1 to 6 ) on their final report card last year (i.e., the grade before the survey). The interestingness measure (i.e., a Wald test statistic over the 20 indirect effects) had a value of $\mathrm{IM}=150.955$. As can be seen in Table 8, only 
two out of 20 indirect effects were significant. There were negative indirect effects of sex and school track on the slope of math compentency development mediated by math-specific self-concept.

The third subgroup consisted of $N=692$ students who were completely satisfied with their health and had a final math grade of satisfactory (i.e., 3 on a scale from 1 to 6 ) on their final report card last year (i.e., the grade before the survey). The interestingness measure (i.e., a Wald test statistic over the 20 indirect effects) had a value of $\mathrm{IM}=148.118$. As can be seen in Table 8, four out of 20 indirect effects were significant. There was a positive indirect effect of socioeconomic status and a negative indirect effect of special educational needs on the intercept of math compentency development mediated by global self-esteem. However, there was also a negative indirect effect of socioeconomic status and a positive indirect effect of special educational needs on the slope of math compentency development mediated by global self-esteem.

\section{Table 9}

Cross-tabulated membership in the three top subgroups indicating degree of overlap

\begin{tabular}{lr}
\hline Membership & $N$ \\
\hline no subgroup & 3392 \\
only subgroup 1 & 101 \\
only subgroup 2 & 104 \\
only subgroup 3 & 146 \\
subgroups 1 \& 2 & 124 \\
subgroups 1 \& 3 & 169 \\
subgroups 2 \& 3 & 163 \\
all subgroups & 158 \\
\hline
\end{tabular}

All three top subgroups are actually further specifications of students with a final grade of satisfactory in the year before the survey. Table 9 gives an overview of the overlap between these groups. In each of the top three subgroups, roughly one sixth is uniquely a member of this subgroup and $N=158$ students are simultaneously a member of all three subgroups. In a post-hoc analysis, we investigated the interestingness measure for the more generalized category (i.e., students with a satisfactory final math grade), which contains 
$N=1272$ student: 3 . The Wald test statistic for this group was IM $=101.634$, which is considerably smaller (i.e., less interesting) than the three specializations reported above.

This illustrates quite well the use of SubgroupSEM for applied settings: the indirect effects vary in specific subgroups and we would not find these patterns in an overall analysis. While it is important to caution that such findings are exploratory and require further empirical validation, they could also serve as potential starting points for further research and the formulation of more in-depth hypotheses.

\section{Discussion}

In this paper, we presented the SubgroupSEM framework, which brings exceptional model mining to structural equation models, enabling an exploratory search for groups with distinct sets of parameters in SEM. SubgroupSEM is a four-step approach in which (a) a two-group structural equation model is specified, (b) a covariate space containing potential group descriptions is chosen, (c) the interestingness of a group is defined as a measure computed from parameters or statistics of interest, and (d) an appropriate subgroup discovery algorithm or heuristic is applied to extract interesting subgroups. We gave two applied examples based on our open-source implementation subgroupsem in $R$ to illustrate the use of our approach.

The SubgroupSEM approach is meant for exploratory and interactive searches for interesting subgroups in order to inform hypothesis generation for confirmatory multi-group analysis. It allows researchers to seek out groups with exceptional sets of parameters in a data-driven manner, while balancing the trade-off between precision and interpretability of the results. In this regard, SubgroupSEM differs from related approaches, like SEMTree, which have a pronounced focus on precise prediction, but do not balance this out with a focus on easily understandable group descriptions and order of interestingness. However, this also emphasizes that SubgroupSEM produces exploratory

\footnotetext{
${ }^{3}$ The interestingness measure was obviously already computed within the subgroup discovery task, however, as only the top $k$ subgroups are returned, we do not obtain every interestingness measure directly.
} 
findings that can help on the investigation of potentially heterogeneous groups in large datasets - it does not relieve the need to confirm such findings in subsequent studies.

\section{Limitations and Extensions}

A crucial factor for efficient, yet exhaustive subgroup discovery is the derivation of optimistic estimates. While we are not aware of any previously derived optimistic estimates directly applicable to SubgroupSEM, this might be a promising avenue for future research. Lemmerich et al. (2016) give an overview of several mean-based, variance based, median-based, rank-based, and distribution-based interestingness measures for numeric target concepts; they further delineate under which circumstances optimistic estimates can be generated for these measures. However, Grosskreutz et al. (2008) point out that formulas for optimistic estimates should themselves not be too computationally demanding and suggest the development of approximative bounds.

We introduced the possibility of obtaining partly overlapping subgroups as a feature of subgroup discovery, which can be handy to explore different combinations of covariates without requiring exclusive group memberships. However, in some cases this can lead to strange results. For example, for a binary variable, both values are often returned as an interesting pattern, or when a specialization contains a large proportion of its generalized category, both are returned (e.g., women under 50, and women). To avoid redundancy in the results set, i.e., the occurrence of many similar subgroups, interestingness measures might be adapted with generalization-aware modifications (Grosskreutz, Boley, \& Krause-Traudes, 2010; Lemmerich et al., 2020). The formulation of these modifications, however, would have to be adapted to the structural equation modeling framework and the interestingness measure at hand. Alternatively, algorithms that promote diverse results can be applied (e.g., van Leeuwen \& Knobbe, 2012)

While we avoid the use of statistical tests during the selection procedure by using test statistics independently of their assumed distribution under the null hypothesis, it might still be tempting to interpret the statistical significance of the resulting subgroup 
models in a conventional way. It might be obvious that the $p$-values of the selected subgroup models (for example, those Table 8 are too liberal as they do not account for the selection procedure. However, Goeman and Solari (2011) argue that most multiple testing methods, like familywise error-based methods (e.g., Bonferroni correction or Romano \& Wolf, 2007) or the false discovery rate (Benjamini \& Hochberg, 1995) are "ill-suited for specific requirements of exploratory research" (p. 584). Instead, they suggest a procedure in which the researcher freely selects the subgroups or hypotheses of greatest interest and then receives a confidence statement on how many false rejections might be included. This approach is available through the $\mathrm{R}$ package cherry and could be combined with SubgroupSEM to assess the risk of false discoveries within the final set of subgroups. 


\section{References}

Ammerman, B. A., Jacobucci, R., \& McCloskey, M. S. (2019). Reconsidering important outcomes of the nonsuicidal self-injury disorder diagnostic criterion A. Journal of Clinical Psychology, 75(6), 1084-1097. doi: 10.1002/jclp.22754

Arminger, G., Stein, P., \& Wittenberg, J. (1999). Mixtures of conditional mean- and covariance-structure models. Psychometrika, 64(4), 475-494. doi: $10.1007 / \mathrm{BF} 02294568$

Benjamini, Y., \& Hochberg, Y. (1995). Controlling the false discovery rate: A practical and powerful approach to multiple testing. Journal of the Royal Statistical Society: Series B (Methodological), 57(1), 289-300. doi: 10.1111/j.2517-6161.1995.tb02031.x

Blossfeld, H.-P., von Maurice, J., Bayer, M., \& Skopek, J. (Eds.). (2016). Methodological Issues of Longitudinal Surveys. Wiesbaden, Germany: Springer. doi: 10.1007/978-3-658-11994-2

Bollen, K. A. (1989). Structural Equations with Latent Variables. Hoboken, NJ, USA: John Wiley \& Sons, Inc. doi: 10.1002/9781118619179

Brandmaier, A. M., Prindle, J. J., McArdle, J. J., \& Lindenberger, U. (2016). Theory-guided exploration with structural equation model forests. Psychological Methods, 21(4), 566-582. doi: 10.1037/met0000090

Brandmaier, A. M., Ram, N., Wagner, G. G., \& Gerstorf, D. (2017). Terminal decline in well-being: The role of multi-indicator constellations of physical health and psychosocial correlates. Developmental Psychology, 53(5), 996-1012. doi: $10.1037 / \operatorname{dev} 0000274$

Brandmaier, A. M., von Oertzen, T., McArdle, J. J., \& Lindenberger, U. (2013). Structural equation model trees. Psychological Methods, 18(1), 71-86. doi: 10.1037/a0030001

Browne, M. W., \& Arminger, G. (1995). Specification and estimation of mean- and covariance-structure models. In G. Arminger, C. C. Clogg, \& M. E. Sobel (Eds.), Handbook of Statistical Modeling for the Social and Behavioral Sciences (pp. 
185-249). Boston, MA: Springer US. doi: 10.1007/978-1-4899-1292-3_4

Carmona, C. J., Gonzalez, P., del Jesus, M. J., \& Herrera, F. (2010). NMEEF-SD:

Non-dominated multiobjective evolutionary algorithm for extracting fuzzy rules in subgroup discovery. IEEE Transactions on Fuzzy Systems, 18(5), 958-970. doi: 10.1109/TFUZZ.2010.2060200

Clark, P., \& Niblett, T. (1989). The CN2 induction algorithm. Machine Learning, 3(4), 261-283. doi: 10.1007/BF00116835

Collins, L. M., \& Lanza, S. T. (2013). Latent class and latent transition analysis: With applications in the social, behavioral, and health sciences. Hoboken, N.J.: Wiley.

Collins, L. M., Schafer, J. L., \& Kam, C.-M. (2001). A comparison of inclusive and restrictive strategies in modern missing data procedures. Psychological Methods, 6(4), 330-351. doi: 10.1037/1082-989X.6.4.330

de Mooij, S. M., Henson, R. N., Waldorp, L. J., \& Kievit, R. A. (2018). Age differentiation within gray matter, white matter, and between memory and white matter in an adult life span cohort. The Journal of Neuroscience, 38(25), 5826-5836. doi: 10.1523/JNEUROSCI.1627-17.2018

del Jesus, M., Gonzalez, P., Herrera, F., \& Mesonero, M. (2007). Evolutionary fuzzy rule induction process for subgroup discovery: A case study in marketing. IEEE Transactions on Fuzzy Systems, 15(4), 578-592. doi: 10.1109/TFUZZ.2006.890662

DeVries, J. M., Szardenings, C., Doebler, P., \& Gebhardt, M. (2021). Subject-specific self-concept and global self-esteem mediate risk factors for lower competency in mathematics and reading. Social Sciences, 10(1), 11. doi: 10.3390/socsci10010011

Duivesteijn, W., Feelders, A., \& Knobbe, A. (2012). Different slopes for different folks: Mining for exceptional regression models with cook's distance. In Proceedings of the 18th ACM SIGKDD international conference on Knowledge discovery and data mining - KDD '12 (p. 868). Beijing, China: ACM Press. doi: $10.1145 / 2339530.2339668$ 
Duivesteijn, W., Feelders, A. J., \& Knobbe, A. (2016). Exceptional Model Mining: Supervised descriptive local pattern mining with complex target concepts. Data Mining and Knowledge Discovery, 30(1), 47-98. doi: 10.1007/s10618-015-0403-4

Gamberger, D., Lavrač, N., \& Krstačić, G. (2003). Active subgroup mining: A case study in coronary heart disease risk group detection. Artificial Intelligence in Medicine, 28(1), 27-57. doi: 10.1016/S0933-3657(03)00034-4

Goeman, J. J., \& Solari, A. (2011). Multiple testing for exploratory research. Statistical Science, 26(4). doi: 10.1214/11-STS356

Grosskreutz, H., Boley, M., \& Krause-Traudes, M. (2010). Subgroup discovery for election analysis: A case study in descriptive data mining. In B. Pfahringer, G. Holmes, \& A. Hoffmann (Eds.), Discovery Science (Vol. 6332, pp. 57-71). Berlin / Heidelberg, Germany: Springer. doi: 10.1007/978-3-642-16184-1_5

Grosskreutz, H., Rüping, S., \& Wrobel, S. (2008). Tight optimistic estimates for fast subgroup discovery. In W. Daelemans, B. Goethals, \& K. Morik (Eds.), Machine Learning and Knowledge Discovery in Databases (Vol. 5211, pp. 440-456). Berlin / Heidelberg, Germany: Springer. doi: 10.1007/978-3-540-87479-9_47

Hämäläinen, W., \& Webb, G. I. (2019). A tutorial on statistically sound pattern discovery. Data Mining and Knowledge Discovery, 33(2), 325-377. doi: $10.1007 / \mathrm{s} 10618-018-0590-\mathrm{X}$

Heath, J. W., Fu, M. C., \& Jank, W. (2009). New global optimization algorithms for model-based clustering. Computational Statistics $\&$ Data Analysis, 53(12), 3999-4017. doi: 10.1016/j.csda.2009.07.007

Herrera, F., Carmona, C. J., González, P., \& del Jesus, M. J. (2011). An overview on subgroup discovery: Foundations and applications. Knowledge and Information Systems, 29(3), 495-525. doi: 10.1007/s10115-010-0356-2

Hipp, J. R., \& Bauer, D. J. (2006). Local solutions in the estimation of growth mixture models. Psychological Methods, 11(1), 36-53. doi: 10.1037/1082-989X.11.1.36 
Holzinger, K. J., \& Swineford, F. (1939). A study in factor analysis: The stability of a bi-factor solution. Supplementary Educational Monographs, 48, xi + 91-xi +91 .

Hothorn, T., \& Zeileis, A. (2015). Partykit: A modular toolkit for recursive partytioning in R. Journal of Machine Learning Research, 16(118), 3905-3909.

Jedidi, K., Jagpal, H. S., \& DeSarbo, W. S. (1997). STEMM: A general finite mixture structural equation model. Journal of Classification, 14(1), 23-50. doi: $10.1007 / \mathrm{s} 003579900002$

Jöreskog, K. G. (1971). Simultaneous factor analysis in several populations. Psychometrika, 36(4), 409-426. doi: 10.1007/BF02291366

Kavšek, B., Lavrač, N., \& Jovanoski, V. (2003). APRIORI-SD: Adapting association rule learning to subgroup discovery. In G. Goos et al. (Eds.), Advances in Intelligent Data Analysis V (Vol. 2810, pp. 230-241). Berlin / Heidelberg, Germany: Springer. doi: 10.1007/978-3-540-45231-7_22

Kelava, A., Nagengast, B., \& Brandt, H. (2014). A nonlinear structural equation mixture modeling approach for nonnormally distributed latent predictor variables. Structural Equation Modeling: A Multidisciplinary Journal, 21(3), 468-481. doi: 10.1080/10705511.2014.915379

Kliegr, T., Bahník, Š., \& Fürnkranz, J. (2020). Advances in machine learning for the behavioral sciences. American Behavioral Scientist, 64(2), 145-175. doi: $10.1177 / 0002764219859639$

Klösgen, W. (1996). EXPLORA: A multipattern and multistrategy discovery assistant. In U. M. Fayyad (Ed.), Advances in Knowledge Discovery and Data Mining (pp. 249-271).

Lanza, S. T., Tan, X., \& Bray, B. C. (2013). Latent class analysis with distal outcomes: A flexible model-based approach. Structural Equation Modeling: A Multidisciplinary Journal, 20(1), 1-26. doi: 10.1080/10705511.2013.742377

Lee, T., \& Shi, D. (2021). A comparison of full information maximum likelihood and 
multiple imputation in structural equation modeling with missing data. Psychological Methods, 26(4), 466-485. doi: 10.1037/met0000381

Leman, D., Feelders, A., \& Knobbe, A. (2008). Exceptional Model Mining. In W. Daelemans, B. Goethals, \& K. Morik (Eds.), Machine Learning and Knowledge Discovery in Databases (Vol. 5212, pp. 1-16). Berlin / Heidelberg, Germany: Springer. doi: 10.1007/978-3-540-87481-2_1

Lemmerich, F. (2014). Novel Techniques For Subgroup Discovery (Unpublished doctoral dissertation).

Lemmerich, F., Atzmueller, M., \& Puppe, F. (2016, May). Fast exhaustive subgroup discovery with numerical target concepts. Data Mining and Knowledge Discovery, 30(3), 711-762. doi: 10.1007/s10618-015-0436-8

Lemmerich, F., \& Becker, M. (2019). Pysubgroup: Easy-to-use subgroup discovery in Python. In U. Brefeld et al. (Eds.), Machine Learning and Knowledge Discovery in Databases (Vol. 11053, pp. 658-662). Cham: Springer International Publishing. doi: 10.1007/978-3-030-10997-4_46

Lemmerich, F., Kiefer, C., Langenberg, B., Cacho Aboukhalil, J., \& Mayer, A. (2020). Mining exceptional mediation models. In D. Helic, G. Leitner, M. Stettinger, A. Felfernig, \& Z. W. Raś (Eds.), Foundations of Intelligent Systems (Vol. 12117, pp. 318-328). Cham: Springer International Publishing. doi:

10.1007/978-3-030-59491-6_30

Liu, H., \& Song, X. Y. (2018). Bayesian analysis of mixture structural equation models with an unknown number of components. Structural Equation Modeling: A Multidisciplinary Journal, 25(1), 41-55. doi: 10.1080/10705511.2017.1372688

Lubke, G. H., \& Muthén, B. (2005). Investigating population heterogeneity with factor mixture models. Psychological Methods, 10(1), 21-39. doi: 10.1037/1082-989X.10.1.21

Lüdtke, O., Marsh, H. W., Robitzsch, A., Trautwein, U., Asparouhov, T., \& Muthén, B. 
(2008). The multilevel latent covariate model: A new, more reliable approach to group-level effects in contextual studies. Psychological Methods, 13(3), 203-229. doi: $10.1037 / \mathrm{a} 0012869$

MacKinnon, D. P. (2012). Introduction to Statistical Mediation Analysis (Second ed.). New York: Routledge. doi: 10.4324/9780203809556

Mampaey, M., Nijssen, S., Feelders, A., \& Knobbe, A. (2012). Efficient Algorithms for Finding Richer Subgroup Descriptions in Numeric and Nominal Data. In 2012 IEEE 12th International Conference on Data Mining (pp. 499-508). Brussels, Belgium: IEEE. doi: 10.1109/ICDM.2012.117

Mattos, J. B., Silva, E. G., de Mattos Neto, P. S. G., \& Vimieiro, R. (2020). Exceptional survival model mining. In R. Cerri \& R. C. Prati (Eds.), Intelligent Systems (Vol. 12320, pp. 307-321). Cham: Springer International Publishing. doi:

10.1007/978-3-030-61380-8_21

Mayer, A., Kiefer, C., Langenberg, B., \& Lemmerich, F. (2021). Using subgroup discovery and latent growth curve modeling to identify unusual developmental trajectories. In Handbook of Computational Social Science, Volume 2 (First ed., pp. 244-268). London: Routledge. doi: 10.4324/9781003025245-18

McArdle, J. J. (1988). Dynamic but structural equation modeling of repeated measures data. In J. R. Nesselroade \& R. B. Cattell (Eds.), Handbook of Multivariate Experimental Psychology (pp. 561-614). Boston, MA: Springer US. doi: 10.1007/978-1-4613-0893-5_17

McLachlan, G. J., \& Peel, D. (2000). Finite mixture models. New York: Wiley. Mehta, P. D., \& Neale, M. C. (2005). People are variables too: Multilevel structural equations modeling. Psychological Methods, 10(3), 259-284. doi: 10.1037/1082-989X.10.3.259

Meredith, W. (1993). Measurement invariance, factor analysis and factorial invariance. Psychometrika, 58(4), 525-543. doi: 10.1007/BF02294825 
Meredith, W., \& Tisak, J. (1990, March). Latent curve analysis. Psychometrika, 55(1), 107-122. doi: 10.1007/BF02294746

Molenberghs, G., Fitzmaurice, G. M., Kenward, M. G., Tsiatis, A. A., \& Verbeke, G. (Eds.). (2015). Handbook of Missing Data Methodology. Boca Raton: CRC Press, Taylor \& Francis Group.

Morgan, J. N., \& Sonquist, J. A. (1963). Problems in the Analysis of Survey Data, and a Proposal. Journal of the American Statistical Association, 415-434.

Muthén, B. O. (1989). Latent variable modeling in heterogeneous populations. Psychometrika, 54(4), 557-585. doi: 10.1007/BF02296397

Muthén, B. O., \& Muthén, L. K. (2000). Integrating person-centered and variable-centered analyses: Growth mixture modeling With latent trajectory classes. Alcoholism: Clinical and Experimental Research, 24(6), 882-891. doi: 10.1111/j.1530-0277.2000.tb02070.x

Peikert, A., \& Brandmaier, A. M. (2021). A reproducible data analysis workflow. Quantitative and Computational Methods in Behavioral Sciences, 1, e3763. doi: $10.5964 /$ qcmb.3763

Quinlan, J. R. (1986). Induction of decision trees. Machine Learning, 1(1), 81-106. doi: $10.1007 / \mathrm{BF} 00116251$

R Core Team. (2021). R: A language and environment for statistical computing [Manual]. Vienna, Austria.

Romano, J. P., \& Wolf, M. (2007). Control of generalized error rates in multiple testing. The Annals of Statistics, 35(4). doi: 10.1214/009053606000001622

Rosseel, Y. (2012). Lavaan: An R package for structural equation modeling. Journal of Statistical Software, 48(2). doi: 10.18637/jss.v048.i02

Serang, S., Jacobucci, R., Stegmann, G., Brandmaier, A. M., Culianos, D., \& Grimm, K. J. (2021). Mplus trees: Structural equation model trees using Mplus. Structural Equation Modeling: A Multidisciplinary Journal, 28(1), 127-137. doi: 
10.1080/10705511.2020.1726179

Shireman, E., Steinley, D., \& Brusco, M. J. (2016). Local optima in mixture modeling. Multivariate Behavioral Research, 51(4), 466-481. doi: $10.1080 / 00273171.2016 .1160359$

Shireman, E., Steinley, D., \& Brusco, M. J. (2017). Examining the effect of initialization strategies on the performance of Gaussian mixture modeling. Behavior Research Methods, 49(1), 282-293. doi: 10.3758/s13428-015-0697-6

Simpson-Kent, I. L., Fuhrmann, D., Bathelt, J., Achterberg, J., Borgeest, G. S., \& Kievit, R. A. (2020). Neurocognitive reorganization between crystallized intelligence, fluid intelligence and white matter microstructure in two age-heterogeneous developmental cohorts. Developmental Cognitive Neuroscience, 41, 100743. doi: 10.1016/j.dcn.2019.100743

Sörbom, D. (1974). A general method for studying differences in factor means and factor structure between groups. British Journal of Mathematical and Statistical Psychology, 27(2), 229-239. doi: 10.1111/j.2044-8317.1974.tb00543.x

Steinley, D., \& Brusco, M. J. (2011). Evaluating mixture modeling for clustering: Recommendations and cautions. Psychological Methods, 16(1), 63-79. doi: $10.1037 / \mathrm{a} 0022673$

Steyer, R., Mayer, A., Geiser, C., \& Cole, D. A. (2015). A theory of states and traits - revised. Annual Review of Clinical Psychology, 11(1), 71-98. doi: 10.1146/annurev-clinpsy-032813-153719

Strobl, C., Malley, J., \& Tutz, G. (2009). An introduction to recursive partitioning: Rationale, application, and characteristics of classification and regression trees, bagging, and random forests. Psychological Methods, 14(4), 323-348. doi: $10.1037 / \mathrm{a} 0016973$

Trajkovski, I., Zelezny, F., Lavrac, N., \& Tolar, J. (2008). Learning Relational Descriptions of Differentially Expressed Gene Groups. IEEE Transactions on Systems, Man, and 
Cybernetics, Part C (Applications and Reviews), 38(1), 16-25. doi:

10.1109/TSMCC.2007.906059

Ushey, K., Allaire, J., \& Tang, Y. (2021). Reticulate: Interface to 'python' [Manual].

Valmarska, A., Lavrač, N., Fürnkranz, J., \& Robnik-Šikonja, M. (2017). Refinement and selection heuristics in subgroup discovery and classification rule learning. Expert Systems with Applications, 81, 147-162. doi: 10.1016/j.eswa.2017.03.041

van Leeuwen, M., \& Knobbe, A. (2012). Diverse subgroup set discovery. Data Mining and Knowledge Discovery, 25(2), 208-242. doi: 10.1007/s10618-012-0273-y

Webb, N. M. (1995). Group collaboration in assessment: Multiple objectives, processes, and outcomes. Educational Evaluation and Policy Analysis, 17(2), 239-261. doi: $10.3102 / 01623737017002239$

Wrobel, S. (1997). An algorithm for multi-relational discovery of subgroups. In J. G. Carbonell et al. (Eds.), Principles of Data Mining and Knowledge Discovery (Vol. 1263, pp. 78-87). Berlin / Heidelberg, Germany: Springer. doi:

10.1007/3-540-63223-9_108

Zeileis, A., Hothorn, T., \& Hornik, K. (2008). Model-based recursive partitioning. Journal of Computational and Graphical Statistics, 17(2), 492-514. doi: $10.1198 / 106186008 X 319331$ 


\section{Appendix A}

\section{Test Statistic-Based Interestingness Measures}

Within the SubgroupSEM package, we provide easy-to-use functions that define an interestingness measure based on a likelihood ratio test or a Wald test respectively. In the following, we describe the computational details of these test statistics and what kind of questions can be addressed with such interestingness measures.

\section{Likelihood Ratio Test-Based Interestingness}

The intuition of a likelihood ratio test is to fit a baseline model for the full dataset, obtaining a baseline log-likelihood value. Then, we proceed with the two-group structural equation models (i.e., modeling for the subgroup and its complement) and compare the log-likelihood values of the two-group models to the log-likelihood of the baseline model. The resulting likelihood ratio expresses how much more likely the data was generated from the two-group model than from the baseline model, or put differently, how much heterogeneity is explained by the distinction between the subgroup and its complement.

More formally, the full model with estimated parameter vector $\hat{\boldsymbol{\theta}}_{\text {restricted }}$ is a restricted version of the two-group model with estimated parameter vector $\hat{\boldsymbol{\theta}}_{\text {unrestricted. }}$. The likelihood ratio test statistic is computed by (cf. Bollen, 1989, p. 292)

$$
\begin{aligned}
L R & =-2\left[\log L\left(\hat{\boldsymbol{\theta}}_{\text {restricted }}\right)-\log L\left(\hat{\boldsymbol{\theta}}_{\text {unrestricted }}\right)\right] \\
& =-2 \log \left[\frac{L\left(\hat{\boldsymbol{\theta}}_{\text {restricted }}\right)}{L\left(\hat{\boldsymbol{\theta}}_{\text {unrestricted }}\right)}\right]
\end{aligned}
$$

where $L(\hat{\boldsymbol{\theta}})$ denotes the likelihood function evaluated at the estimated parameters of the baseline or two-group model respectively. The aforementioned ratio of likelihoods can be seen in Equation (A1). If using the LR test statistic for a single hypothesis test, it can be shown that it has a limiting $\chi^{2}$ distribution, for which the degrees of freedom are equal to the difference in degrees of freedom between the two models (cf. Bollen, 1989). However, these properties usually do not transfer to multiple testing scenarios, such as subgroup 
discovery tasks (Hämäläinen \& Webb, 2019).

In the SubgroupSEM approach, the LR is itself used as an interestingness measure, as it already provides useful information on the proportional gain in likelihood of the data given the two-group model compared to the baseline model. Translated into an interestingness measure, LR allows for searching for subgroups which maximize the likelihood of the dataset at hand. This approach of using the proportional gain in the likelihood rather than the associated $p$-value has recently been suggested for Mplus trees (Serang et al., 2021) as well. This interestingness measure evaluates the total amount of heterogeneity between subgroup and complement. For a more fine-grained interestingness measure, focusing on particular parts of a model, Wald test-based interestingness measures can be useful.

\section{Wald Test-Based Interestingness}

The intuition of a Wald test-based interestingness measures is to formulate equality constraints for particular parameters of interests in both groups (e.g., equal means in both groups, $\mu_{0}=\mu_{1}$ ) and then test how much the estimated parameters deviate from these imposed constraints. Translated into an interestingness measure, such an test statistic answers the question "To what extent are the parameters of interest statistically not equal between groups?".

More formally, we formulate a vector-valued function with restrictions on the parameters of the unconstrained two-group model $\mathbf{r}(\boldsymbol{\theta})$. Then the Wald test statistic is Bollen, 1989):

$$
\mathrm{W}=\mathbf{r}(\hat{\boldsymbol{\theta}})^{\mathrm{T}}\left(\left[J_{\mathbf{r}}(\hat{\boldsymbol{\theta}})\right]^{\mathrm{T}} \boldsymbol{\Sigma}_{\hat{\boldsymbol{\theta}}} J_{\mathbf{r}}(\hat{\boldsymbol{\theta}})\right) \mathbf{r}(\hat{\boldsymbol{\theta}})
$$

where $J_{\mathbf{r}}(\hat{\boldsymbol{\theta}})$ is the Jacobian matrix of the restriction function and $\boldsymbol{\Sigma}_{\hat{\boldsymbol{\theta}}}$ is the covariance matrix of the estimated parameters. For example, when $\mathbf{r}(\boldsymbol{\theta})$ consists of a single constraint 
of $\theta_{1}=0, \mathrm{~W}$ is

$$
\mathrm{W}=\frac{\hat{\theta}_{1}^{2}}{\operatorname{avar}\left(\hat{\theta}_{1}\right)}
$$

where $\operatorname{avar}\left(\hat{\theta}_{1}\right)$ is the estimated asymptotic variance of $\hat{\theta}_{1}$ (example from Bollen, 1989, p. 294). An advantage of this procedure is, that we do not look at the raw deviation of $\hat{\theta}_{1}$ from zero, but take the estimation uncertainty into account. This allows us to test multiple constraints on parameters of interest simultaneously, which is more targeted than the likelihood ratio test, but still provides information about whether a subgroup is statistically interesting with regard to multiple parameters.

Again, if we were to use the Wald test statistic $W$ in regular hypothesis testing, it would follow a $\chi^{2}$ distribution with degrees of freedom equal to the number of equality constraints. As mentioned above, these properties do not transfer into the world of subgroup discovery with its exploratory, multiple tests. We will cover this issue in the next paragraphs. 


\section{Appendix B}

\section{Multiple Testing}

The issue of multiple testing refers to the problem that the properties of most statistical tests are derived under the assumption of testing a single hypothesis once. The corresponding Type 1 error rate (i.e., the probability of obtaining a false positive significant result) does not hold if the same test is repeated over and over for a number of estimated parameters. For example, if we evaluate a two-group model using a likelihood ratio test as presented before, the nominal Type 1 error rate of $5 \%$ would indicate, that in 1 out of 20 cases, we would falsely detect a significant difference between the baseline and the two-group model. However, if we repeatedly applied a likelihood ratio test to compare hundreds of subgroup models to a baseline model, the overall chance that at least one false positive difference would be detected is decidedly higher than $5 \%$.

In SubgroupSEM, the issue of multiple testing must be considered at two different stages of the subgroup discovery task: First, when the $p$-value of an actual statistical test is used to define an interestingness measure, for example, the $p$-value of a likelihood ratio test. In this scenario, subgroups with the smallest $p$-value could be defined as most interesting. It is important to note that the $p$ values should be interpreted with caution, as they do not have their classical meaning due to Type 1 error accumulation. In SubgroupSEM we avoid these interpretative issues by focusing on the test statistics rather than the $p$ values. If, however, researchers wish to base subgroup discovery on $p$-values and want to properly interpret them, some kind of correction for multiple testing should be applied. For an overview of correction methods within the field of pattern mining, see Hämäläinen and Webb (2019). Second, when inspecting and evaluating the model fit and estimated parameters of the final subgroup models, the uncorrected $p$-values are not

necessarily trustworthy. This was illustrated, for example, by Goeman and Solari (2011) for covariate selection algorithms in regression models. However, Goeman and Solari argue that classical corrections for multiple testing are "contrary to the spirit of exploratory 
research" (p.595), as exploratory research seeks to generate potential hypotheses for further investigation knowing that some of these suggested hypotheses might turn out to be false positives. Instead, they propose an alternative approach for multiple testing within exploratory research, which we discuss in the concluding section of the paper. 